\title{
Reevaluating the Role of the Saharan Air Layer in Atlantic Tropical Cyclogenesis and Evolution
}

\author{
SCOTT A. BRAUN \\ Laboratory for Atmospheres, NASA Goddard Space Flight Center, Greenbelt, Maryland
}

(Manuscript received 7 July 2009, in final form 17 December 2009)

\begin{abstract}
The existence of the Saharan air layer (SAL), a layer of warm, dry, dusty air frequently present over the tropical Atlantic Ocean, has long been appreciated. The nature of its impacts on hurricanes remains unclear, with some researchers arguing that the SAL amplifies hurricane development and with others arguing that it inhibits it. The potential negative impacts of the SAL include 1) vertical wind shear associated with the African easterly jet; 2) warm air aloft, which increases thermodynamic stability at the base of the SAL; and 3) dry air, which produces cold downdrafts. Multiple NASA satellite datasets and NCEP global analyses are used to characterize the SAL's properties and evolution in relation to tropical cyclones and to evaluate these potential negative influences. The SAL is shown to occur in a large-scale environment that is already characteristically dry as a result of large-scale subsidence. Strong surface heating and deep dry convective mixing enhance the dryness at low levels (primarily below $\sim 700 \mathrm{hPa}$ ), but moisten the air at midlevels. Therefore, mid- to-upper-level dryness is not generally a defining characteristic of the SAL, but is instead often a signature of subsidence. The results further show that storms generally form on the southern side of the jet, where the background cyclonic vorticity is high. Based upon its depiction in NCEP Global Forecast System meteorological analyses, the jet often helps to form the northern side of the storms and is present to equal extents for both strengthening and weakening storms, suggesting that jet-induced vertical wind shear may not be a frequent negative influence. Warm SAL air is confined to regions north of the jet and generally does not impact the tropical cyclone precipitation south of the jet.

Composite analyses of the early stages of tropical cyclones occurring in association with the SAL support the inferences from the individual cases noted above. Furthermore, separate composites for strongly strengthening and for weakening storms show few substantial differences in the SAL characteristics between these two groups, suggesting that the SAL is not a determinant of whether a storm will intensify or weaken in the days after formation. Key differences between these cases are found mainly at upper levels where the flow over strengthening storms allows for an expansive outflow and produces little vertical shear, while for weakening storms, the shear is stronger and the outflow is significantly constrained.
\end{abstract}

\section{Introduction}

Synoptic outbreaks of Saharan dust occur from late spring to early fall and can extend from western Africa across the tropical Atlantic Ocean to the Caribbean (Prospero et al. 1970; Prospero and Carlson 1970, 1972). The dust is carried predominantly westward within the Saharan air layer (SAL), which is formed by strong surface heating as westward-moving air crosses the Saharan desert. The heating produces a deep well-mixed layer with warm temperatures and low relative humidity $(\mathrm{RH})$

Corresponding author address: Dr. Scott A. Braun, NASA GSFC, Mail Code 613.1, Greenbelt, MD 20771.

E-mail: scott.a.braun@nasa.gov at low levels. As the warm, dry air moves off the African coast, it is undercut by cooler, moister air to form the elevated SAL (Karyampudi and Carlson 1988). The vertical thermodynamic structure over the Atlantic consists of a well-mixed marine boundary layer capped by the trade wind inversion near $850 \mathrm{hPa}$, where the SAL begins (Carlson and Prospero 1972; Diaz et al. 1976; Prospero and Carlson 1981; Karyampudi and Carlson 1988; Karyampudi et al. 1999; Karyampudi and Pierce 2002). The SAL extends from $\sim 800$ to $550 \mathrm{hPa}$ near the coast of Africa and is characterized by nearly constant potential temperature and vapor mixing ratio (Carlson and Prospero 1972; Karyampudi and Carlson 1988). The base of the SAL rises while the top of the SAL slowly sinks to the west. Temperatures near the top of the SAL 
tend to be somewhat cooler than the surrounding tropical atmosphere so that the SAL is typically capped by another inversion (Carlson and Prospero 1972).

The strong horizontal temperature gradients along the leading and southern borders of the SAL give rise to a maximum in the geostrophic wind (due to thermal wind considerations) to produce the midlevel African easterly jet (AEJ) along the southern edge of the SAL (Karyampudi and Carlson 1988; Cook 1999). This jet is associated with large vertical and horizontal wind shears and an ageostrophic transverse circulation that enhances upward motion in the dust-free air to the south of the jet, leading to deep convection there, and downward motion within the SAL (Karyampudi and Carlson 1988; Karyampudi et al. 1999; Karyampudi and Pierce 2002).

The impacts of the SAL on the development of tropical cyclones are not well understood. Early studies (e.g., Karyampudi and Carlson 1988; Karyampudi and Pierce 2002) suggested a potential positive influence on the growth of easterly waves and tropical cyclones in the Atlantic. A more recent study by Dunion and Velden (2004, hereafter DV) described several potentially negative influences of the SAL, and Wu et al. (2006), Jones et al. (2007), and Shu and Wu (2009) assumed the general applicability of the DV results to describe SAL impacts on hurricanes. The reduced Atlantic hurricane activity of 2006 and 2007 compared to 2004 and 2005, particularly as it affected the United States, has led to speculation in some research papers (e.g., Lau and Kim 2007a,b; Sun et al. 2008) that dustiness or dry air from increased SAL activity contributed significantly to the decline in hurricane activity in those two years. Wu (2007) related recent increased Atlantic hurricane activity to a decrease in SAL activity (dry-air outbreaks and enhanced vertical wind shear). But is this focus on the negative impacts of the SAL warranted? Is the SAL a major influence, just one of many factors, or is it only a minor influence on Atlantic hurricane activity?

Karyampudi and Carlson (1988) and Karyampudi and Pierce (2002) suggested that the SAL contributes to easterly wave growth and, in some cases tropical cyclogenesis, by supporting convection along its leading and southern borders. The SAL increases the strength of the AEJ and its associated vorticity patterns. The AEJ leads to weak cyclonic or even anticyclonic potential vorticity (PV) north of the jet, strong positive PV south of the jet, and a significant PV-gradient sign reversal. The latter favors easterly wave growth via barotropic instability. Karyampudi and Carlson (1988) also showed that the baroclinic aspects of the AEJ, via the induced ageostrophic circulation and attendant convection, also contribute to wave growth. Thus, Karyampudi and Carlson (1988) and Karyampudi and Pierce (2002) conclude that the SAL can aid wave growth and tropical cyclone development. Jones et al. (2004) presented indirect evidence of the impacts of African dust on easterly waves by comparing analysis increments of geopotential height using 22 yr of National Centers for Environmental PredictionNational Centers for Atmospheric Research (NCEPNCAR) reanalysis data. They found larger amplitudes in the analysis than in the first guess, suggesting amplification of easterly waves via the radiative effects of dust, which were incorporated into the reanalysis by data assimilation, but were absent in the model first guess.

In contrast, DV focused on mechanisms that generally inhibit tropical cyclone genesis and intensification. They suggested that the SAL negatively impacts tropical cyclones in the following ways: 1) the enhanced low-level temperature inversion, maintained by radiative warming of dust, suppresses deep convective development; 2) vertical wind shear caused by an increase in the lowlevel easterlies associated with the AEJ inhibits tropical cyclone intensification, based upon studies that have shown that shear tends to weaken storms (Gray 1968; Merrill 1988; DeMaria and Kaplan 1994, 1999; Frank and Ritchie 2001; Rogers et al. 2003; Braun and Wu 2007); and 3) intrusions of dry SAL air into tropical cyclones foster enhanced cold downdrafts (Emanuel 1989; Powell 1990) and lower the convective available potential energy within tropical cyclones. While it was not Dunion and Velden's intention to imply that the SAL's impacts were always negative or were the dominant factor affecting hurricane activity (J. Dunion and C. Velden 2009, personal communication), it appears from recent research papers (e.g., Jones et al. 2007; Wu 2007; Sun et al. 2008, 2009; Reale et al. 2009; Shu and Wu 2009) that that view is becoming more widespread.

The effects of Saharan dust will not be a major focus of the present paper. Dust is thought to potentially impact storms in two ways: through effects on the cloud microphysics within the storms and through absorption of incoming solar radiation, which warms the low- to midlevel air mass and reduces the incident solar radiation at the ocean surface. Studies of the impacts of dust on cloud microphysics in convective systems and hurricanes have been inconclusive regarding whether dust would enhance or weaken convection and hurricanes (Zhang et al. 2007; Tao et al. 2007; Jenkins and Pratt 2008; Jenkins et al. 2008). Several studies have shown a correlation between dust activity, sea surface temperature (SST), and tropical cyclone activity, with higher dust activity associated with cooler SSTs and fewer hurricanes (Lau and Kim 2007a,b; Evan et al. 2007, 2008). While a direct causal relationship between dust and SSTs exists because of the impacts of dust on solar insolation at the ocean surface, the causality implied by the relationship with storm activity is less clear 
since the dust-induced SST cooling is only a small fraction of a degree and is just one of many influences on tropical cyclone activity.

This study evaluates the negative impacts of the SAL as described by DV. The link between the SAL's properties and its supposed negative impacts (DV; Wu 2007; Shu and Wu 2009; Sun et al. 2008) has been built largely on very limited evidence and some false assumptions. Alternative causes of storm weakening or lack of intensification, such as vertical shear or weak convective activity not associated with the SAL, and hurricane-induced ocean cooling, were not examined. Here, we reevaluate the extent to which the SAL acts as a negative influence on individual storms. The paper is organized as follows. Section 2 describes the datasets used and the analysis methods. Section 3 provides a climatic context for the SAL by examining the mean properties of the SAL and its environment over the Atlantic and African regions. Particular attention is given to the relative humidity distributions within the SAL and its nonSAL environment. Section 4 discusses the role of vertical shear and the warm SAL air (i.e., high stability). Section 5 describes composite environmental properties within a storm-centered reference frame and evaluates the mean structure for both strengthening and weakening storms. Sections 6 and 7 provide discussion and conclusions.

\section{Data}

Several National Aeronautics and Space Administration (NASA) satellites currently provide information that is critical to assessing the impacts of the SAL on hurricanes. The Tropical Rainfall Measuring Mission (TRMM) provides information on the rainfall amount and structure in tropical systems. Here, we use the TRMM multisatellite precipitation product [known as the TRMM $3 \mathrm{~B} 42$ product; Huffman et al. (2007)], which provides rain-rate estimates every $3 \mathrm{~h}$. The Moderate Resolution Imaging Spectroradiometer (MODIS) imager, on both the Aqua and Terra satellites since 2002, provides a measure of the vertically integrated dust concentration, or aerosol optical depth (AOD), within the SAL. The Cloud-Aerosol Lidar and Infrared Pathfinder Satellite Observations (CALIPSO) satellite provides accurate estimates of cloud and aerosollayer occurrence, their top and base heights, and optical depths. The Atmospheric Infrared Sounder (AIRS) and Advanced Microwave Sounding Unit (AMSU) retrieve temperature and humidity profiles that are essential to characterizing the thermodynamic properties of the SAL. All data sources are summarized in Table 1.

CALIPSO data for June-August (JJA) 2006-07 are used in this study to characterize summer dust distributions. The horizontal distribution of dust-layer frequency is computed as the number of CALIPSO-identified dust layers ${ }^{1}$ (from the $80-\mathrm{km}$ average product to improve dust detection) occurring within $5^{\circ}$-wide boxes divided by the total number of CALIPSO observations within the same boxes, multiplied by 100 . Note that the total number of CALIPSO observations includes occurrences of overlying clouds that prevent detection of dust layers below. As a result, the dust frequencies in some locations (particularly near the African coast) may be too low. In this regard, the frequencies shown represent the frequency of detection by CALIPSO rather than the true frequency of occurrence. The vertical distribution of the dust frequency is determined similarly by using the aerosol-layer base and top heights (including multiple layers) to define occurrence on a grid with $5^{\circ}$ horizontal and 500-m vertical spacing.

Of particular note regarding the AIRS-AMSU data is that the temperature data for a particular pressure level is the temperature at that level while the relative humidity for a specified level is the layer-averaged RH from the specified level to the next level above. For example, AIRS $\mathrm{RH}$ data at $850 \mathrm{hPa}(700 \mathrm{hPa})$ is the average over the layer from 850 to $700 \mathrm{hPa}(700$ to $600 \mathrm{hPa}$ ). Daily analyses of MODIS AODs are created by combining Terra and Aqua MODIS data as follows: for grid points with no valid data for a given day, the grid point is assigned a missing value; for one valid data value, the value is taken as the mean value; for two valid data values, the average of the values is used. Monthly average values of AIRS-AMSU relative humidity and temperature are obtained from the level-3 monthly mean standard product. Daily averages are obtained that are similar to those from MODIS except using twice-a-day observations from AIRS-AMSU.

Since satellite data provide limited wind information, NCEP Global Forecasting System (GFS) final global analyses, available every $6 \mathrm{~h}$, are used to characterize the kinematic properties of the SAL and its environment. In section 5, the GFS data are used to construct composite fields of the horizontal and vertical winds, temperature, relative humidity, convective available potential energy (CAPE), convective inhibition (CIN), and convective cloud fraction in a reference frame centered on the storms. Storms were selected based upon the general criteria that they form in association with African easterly waves (AEWs) and along SAL outbreaks. These conditions were typically met by all storms forming east of $70^{\circ} \mathrm{W}$ and south of $25^{\circ} \mathrm{N}$. Storms forming farther westward or northward were far less likely to have significant SAL interactions. Forty-one storms from 2001

\footnotetext{
${ }^{1}$ We exclude the CALIPSO “polluted dust" category because of uncertainties about what fraction represents mineral dust versus other aerosol types. If more than one dust layer is identified, only the first is counted.
} 
TABLE 1. Summary of the data used in this study.

\begin{tabular}{|c|c|c|c|c|}
\hline Dataset & Measurement & Horizontal resolution & Frequency (h) & Description \\
\hline TRMM & Rainfall rate $\left(\mathrm{mm} \mathrm{h}^{-1}\right)$ & $0.25^{\circ}$ & 3 & $\begin{array}{l}\text { TRMM multisatellite precipitation } \\
\text { product (3B42; Huffman et al. } 2007 \text { ) }\end{array}$ \\
\hline MODIS & AOD & $\begin{array}{l}1^{\circ} \text { for level } 3,10 \mathrm{~km} \\
\text { for level } 2\end{array}$ & 24 & $\begin{array}{l}\text { MODIS level } 2 \text { and } 3 \text { products } \\
\text { (Salomonson et al. 1989) }\end{array}$ \\
\hline AIRS-AMSU & Temperature, $\mathrm{RH}$ profiles & $\begin{array}{l}1^{\circ} \text { for level } 3,45 \mathrm{~km} \\
\text { for level } 2\end{array}$ & 12 & $\begin{array}{l}\text { AIRS level } 2 \text { and } 3 \text { products; } 13 \\
\text { vertical levels in the troposphere } \\
\text { (Aumann et al. 2003) }\end{array}$ \\
\hline NCEP & $3 \mathrm{D}$ winds, temperature, $\mathrm{RH}$ & $1^{\circ}$ & 6 & $\begin{array}{l}\text { NCEP final GFS analyses archived } \\
\text { at NCAR }\end{array}$ \\
\hline
\end{tabular}

to 2008 were found to meet these criteria and are listed in Table 2. Composite fields were obtained for the time (designated $t_{0}$ ) when the storms were first identified in the Hurricane Data (HURDAT) archive (information online at http://www.aoml.noaa.gov/hrd/hurdat/) for 200107 or in best-track summaries for 2008 systems that were also available from the National Oceanic and Atmospheric Administration/Hurricane Research Division (NOAA/ HRD) Web site. Note that tropical depressions that did not intensify further and were not listed in the HURDAT record or on the HRD Web site have not been included in this analysis. Composite fields were also computed for days $t_{0-2}$ through $t_{0+4}$ (only results through $t_{0+2}$ are shown herein), where the subscript indicates the time, in days, relative to $t_{0}$. For days prior to $t_{0}$, when possible, the storm center position was estimated using the GFS vorticity and wind fields averaged between 950 and $600 \mathrm{hPa}$, as well as TRMM data. Reliable estimates of storm position at $t_{0-2}$ were not available for Danielle (2004) and Debbie (2006) and so have been excluded from the composite for that time. For each storm and each time, the above-mentioned fields were obtained for a $60^{\circ}$ latitude by $60^{\circ}$ longitude box centered on the storm. Composite fields were then obtained by averaging data for all storms over these boxes for each time. Furthermore, in an effort to distinguish between characteristics of storms that intensify in their early stages and those that weaken, composite fields were obtained for subsets of the total number of storms. For intensifying events, storms were required to increase in intensity by at least $20 \mathrm{kt}\left(10.3 \mathrm{~m} \mathrm{~s}^{-1}\right)$ between days 2 and 4 after initiation. For weakening cases, storms were required to weaken by any amount over the same period. The composite fields for intensifying storms were obtained from 18 cases, while the composite for weakening storms came from 13 cases (see Table 2). The mean storm positions at the time of formation were $13.0^{\circ} \mathrm{N}, 39.9^{\circ} \mathrm{W}$ for strengthening storms and $12.7^{\circ} \mathrm{N}, 44.0^{\circ} \mathrm{W}$ for weakening storms.

The significance of the differences between strengthening and weakening cases was measured in two ways. The first is the Student's $t$ test. The second is a calculation of the correlation at each grid point between the intensity change between days $t_{0+2}$ and $t_{0+4}$ and some parameter of interest such as zonal or meridional wind, relative humidity, etc. Areas of high significance from the Student's $t$ test often coincide with areas of higher correlation, with the latter indicating whether the difference is typically associated with a strengthening or weakening of storms.

The following results must be viewed with several caveats in mind. First, our examination only includes named storm systems. Consequently, the conclusions cannot be applied to nondeveloping tropical disturbances or depressions. Second, because of the lack of detailed wind data over the oceans and the relatively coarse resolution of the NCEP data, the GFS analyses may not capture the magnitude and more detailed structure of the AEJ or the tropical cyclones. As a result, the exact extent to which vertical shear associated with the jet encroaches upon the core of the storms may be difficult to assess. Conclusions from the composite study in section 5 should be viewed with this caveat in mind. However, the qualitative relationships between the AEJ and tropical cyclones should be well described (Tompkins et al. 2005; Sultan et al. 2007). Third, the AIRS-AMSU data and the GFS analyses lack the vertical resolution needed to capture very shallow layers and may therefore underestimate the dryness of the low-level SAL and the strength of the low-level inversion. Although the GFS analyses have their limitations, the comparisons between the GFS-derived characteristics of strengthening and weakening storms are between similar quantities having similar error characteristics. Finally, the AIRS-retrieved profiles of temperature and humidity are likely to be impacted by the radiative effects of dust. However, these impacts have not yet been well characterized. Comparison of the timeaveraged AIRS data to GFS analyses and NASA Modern Era Retrospective Analysis for Research and Applications (MERRA) analyses (not shown) indicates very good consistency in all of the respects discussed in section 3 . While not very well resolved in the vertical, the AIRSAMSU data readily capture the warmth and dryness of 
TABLE 2. Storms used in the SAL composites. Storms included in the "weakening storms" composite are indicated in italics, while those included in the "strengthening storms" composite are indicated in boldface. Day 2 and 4 intensities are the maximum wind speeds 2 and 4 days after the genesis time. Intensity change is the difference between the day 4 and day 2 intensities. Earl dissipated prior to day 4 , so day 4 intensity and intensity change are not available.

\begin{tabular}{|c|c|c|c|c|c|c|}
\hline Storm & Genesis date & $\begin{array}{c}\text { Genesis location } \\
\left({ }^{\circ} \mathrm{N},{ }^{\circ} \mathrm{W}\right)\end{array}$ & $\begin{array}{c}\text { Initial } \\
\text { intensity (kt) }\end{array}$ & $\begin{array}{c}\text { Day } 2 \\
\text { intensity (kt) }\end{array}$ & $\begin{array}{c}\text { Day } 4 \\
\text { intensity (kt) }\end{array}$ & $\begin{array}{l}\text { Intensity } \\
\text { change (kt) }\end{array}$ \\
\hline Chantal & 1800 UTC 14 Aug 2001 & $12.8,37.0$ & 25 & 30 & 50 & $+\mathbf{2 0}$ \\
\hline Erin & 1800 UTC 1 Sep 2001 & $12.5,34.3$ & 30 & 40 & 25 & -15 \\
\hline Felix & 1800 UTC 7 Sep 2001 & $13.9,28.4$ & 30 & 25 & 35 & +10 \\
\hline Dolly & 1200 UTC 29 Aug 2002 & $9.5,31.2$ & 30 & 35 & 45 & +10 \\
\hline Isidore & 1800 UTC 14 Sep 2002 & $10.0,60.5$ & 25 & 20 & 45 & +25 \\
\hline Lili & 1800 UTC 21 Sep 2002 & $10.2,44.6$ & 25 & 50 & 40 & -10 \\
\hline Claudette & 0000 UTC 7 Jul 2003 & $11.1,53.5$ & 25 & 50 & 50 & 0 \\
\hline Fabian & 1800 UTC 27 Aug 2003 & $14.6,30.7$ & 25 & 60 & 115 & +55 \\
\hline Isabel & 0000 UTC 6 Sep 2003 & 13.8, 31.4 & 30 & 80 & 110 & $+\mathbf{3 0}$ \\
\hline Kate & 1800 UTC 25 Sep 2003 & $11.8,37.7$ & 30 & 35 & 65 & $+\mathbf{3 0}$ \\
\hline Bonnie & 1200 UTC 3 Aug 2004 & $12.9,53.6$ & 25 & 25 & 20 & -5 \\
\hline Charley & 1200 UTC 9 Aug 2004 & $11.4,59.2$ & 30 & 60 & 95 & +35 \\
\hline Danielle* & 1200 UTC 13 Aug 2004 & $12.3,21.8$ & 30 & 80 & 90 & +10 \\
\hline Earl & 1800 UTC 13 Aug 2004 & $9.0,45.6$ & 30 & 35 & - & - \\
\hline Frances & 0000 UTC 25 Aug 2004 & 11.1, 35.2 & 25 & 70 & 115 & +45 \\
\hline Ivan & 1800 UTC 2 Sep 2004 & 9.7, 27.6 & 25 & 55 & 90 & +35 \\
\hline Jeanne & 1800 UTC 13 Sep 2004 & $15.9,60.0$ & 25 & 60 & 30 & -30 \\
\hline Karl & 0600 UTC 16 Sep 2004 & $11.2,29.2$ & 30 & 85 & 115 & $+\mathbf{3 0}$ \\
\hline Lisa & 1800 UTC 19 Sep 2004 & $13.3,32.4$ & 30 & 60 & 30 & -30 \\
\hline Dennis & 1800 UTC 4 Jul 2005 & $12.0,60.8$ & 25 & 60 & 120 & +60 \\
\hline Emily & 0000 UTC 11 Jul 2005 & $10.7,42.4$ & 25 & 45 & 110 & +65 \\
\hline Irene & 1800 UTC 4 Aug 2005 & $12.9,33.5$ & 25 & 30 & 30 & 0 \\
\hline Lee & 1200 UTC 28 Aug 2005 & $14.0,45.1$ & 25 & 20 & 30 & +10 \\
\hline Maria & 1200 UTC 1 Sep 2005 & $18.8,45.5$ & 30 & 50 & 85 & +35 \\
\hline Philippe & 1200 UTC 17 Sep 2005 & $13.3,54.5$ & 30 & 65 & 45 & -20 \\
\hline Chris & 0000 UTC 1 Aug 2006 & $16.3,58.1$ & 30 & 50 & 25 & -25 \\
\hline Debby* & 1800 UTC 21 Aug 2006 & $11.6,21.7$ & 30 & 45 & 35 & -10 \\
\hline Ernesto & 1800 UTC 24 Aug 2006 & $12.7,61.6$ & 30 & 55 & 35 & -20 \\
\hline Florence & 1800 UTC 3 Sep 2006 & $14.1,39.4$ & 30 & 40 & 40 & 0 \\
\hline Gordon & 1800 UTC 10 Sep 2006 & $20.2,53.8$ & 25 & 55 & 105 & $+\mathbf{5 0}$ \\
\hline Helene & 1200 UTC 12 Sep 2006 & $11.9,22.0$ & 25 & 40 & 65 & +25 \\
\hline Dean & 0600 UTC 13 Aug 2007 & $12.2,28.9$ & 30 & 50 & 80 & $+\mathbf{3 0}$ \\
\hline Felix & 1200 UTC 31 Aug 2007 & $11.5,56.6$ & 25 & 90 & 140 & $+\mathbf{5 0}$ \\
\hline Ingrid & 0600 UTC 12 Sep 2007 & $13.0,43.6$ & 25 & 35 & 30 & -5 \\
\hline Karen & 0000 UTC 25 Sep 2007 & $10.0,35.9$ & 30 & 60 & 35 & -25 \\
\hline Melissa & 0600 UTC 28 Sep 2007 & $14.0,25.8$ & 25 & 30 & 20 & -10 \\
\hline Bertha & 0600 UTC 3 Jul 2008 & $12.7,22.9$ & 30 & 45 & 65 & $+\mathbf{2 0}$ \\
\hline Fay & 1200 UTC 15 Aug 2008 & $18.4,67.4$ & 30 & 45 & 55 & +10 \\
\hline Gustav & 0000 UTC 25 Aug 2008 & $13.5,67.4$ & 25 & 50 & 60 & +10 \\
\hline Hanna & 0000 UTC 28 Aug 2008 & $19.4,57.3$ & 30 & 45 & 45 & 0 \\
\hline Ike & 0600 UTC 1 Sep 2008 & $17.2,37.0$ & 30 & 55 & 115 & +60 \\
\hline
\end{tabular}

* Position estimates 2 days prior to genesis could not be determined.

the SAL (see section 3a) in horizontal maps, and these qualitative patterns are likely representative.

\section{Basic characteristics of the SAL and the large-scale Atlantic environment}

a. Dust and vertical mixing over the Sahara

Satellite-derived properties of the SAL for JJA 200607 from CALIPSO and JJA 2006 from TRMM are shown in Fig. 1. Dust is most frequently detected over northern Africa and over the eastern Atlantic Ocean, where peak values fall off from $>50 \%$ at the African coast to $<30 \%$ by $\sim 70^{\circ} \mathrm{W}$. Dust is largely confined meridionally between approximately $10^{\circ}$ and $35^{\circ} \mathrm{N}$ latitude over the Atlantic, with peak values at the African coast near $20^{\circ} \mathrm{N}$, dropping southward to $17.5^{\circ} \mathrm{N}$ farther west. Precipitation is largely absent within the dust layer, supporting conclusions that the SAL suppresses moist convection within the air mass (Wong and Dessler 2005). However, this result should 


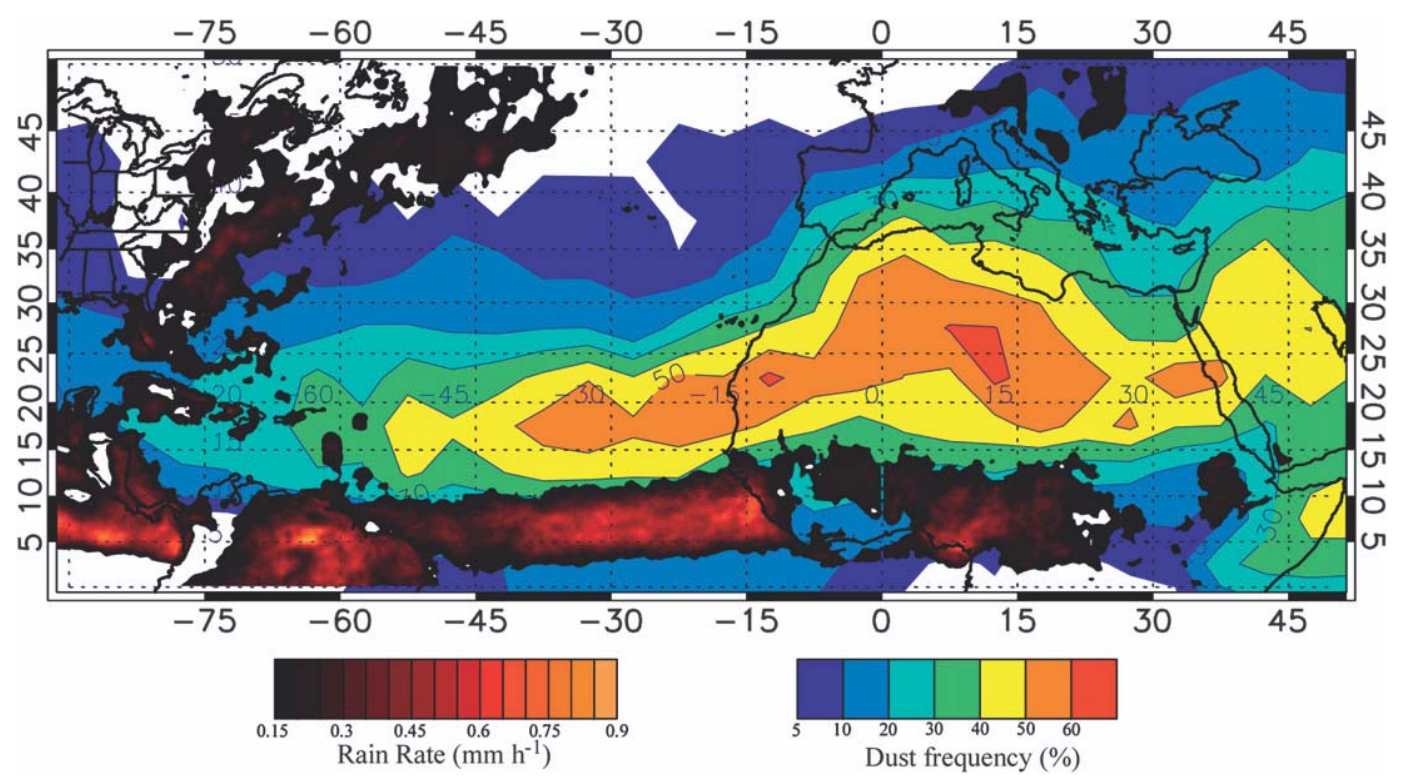

FIG. 1. JJA 2006-07 mean dust detection frequency from CALIPSO and JJA 2006 mean surface rainfall rate from the TRMM multisatellite precipitation product.

not be taken to mean that the SAL suppresses the convection needed for the genesis and intensification of hurricanes since precipitation is abundant south of the southern boundary of the SAL.

The mean vertical structure of the SAL is shown in Fig. 2, in which CALIPSO-derived dust frequencies averaged between $12.5^{\circ}$ and $32.5^{\circ} \mathrm{N}$ are shown along with August 2003-08 averaged temperature perturbations and relative humidity between $18^{\circ}$ and $30^{\circ} \mathrm{N}^{2}$ Over Africa, dust occurs within a deep layer extending upward to $6 \mathrm{~km}$, with peak frequency around $2.5-\mathrm{km}$ altitude. Moving westward from the African coast, dust frequency decreases slowly out to $40^{\circ} \mathrm{W}$ and then more rapidly beyond that longitude. The top of the dust layer drops to just above $4 \mathrm{~km}$ by $70^{\circ} \mathrm{W}$ while the base of the dust layer rises over the cooler marine layer to $\sim 1 \mathrm{~km}$ near $20^{\circ} \mathrm{W}$ and remains there farther westward.

Average temperatures in Fig. 2a are shown as perturbations from the values at the left edge of the diagram at $90^{\circ} \mathrm{W}$. Very warm temperature perturbations are present at low levels over Africa and Saudi Arabia, change to temperatures comparable to downstream (westward) regions by $\sim 5-\mathrm{km}$ altitude, and are slightly cooler than to the west near the top of the dust layer near $6 \mathrm{~km}$. Temperatures at low levels decrease very rapidly offshore to the west, partly as SAL air is undercut by marine air below $\sim 2 \mathrm{~km}$ and also as the SAL cools as a result of net

\footnotetext{
${ }^{2}$ The lower-latitude limit of $18^{\circ} \mathrm{N}$ is chosen to reduce the impacts of passing AEWs and better isolate the characteristics of the SAL.
}

radiative cooling. Relative humidity (Fig. 2b) at low levels over the Sahara decreases to below $30 \%$ as a result of the strong low-level warming. Moist air is found to the west of Africa beneath the dust layer, further suggesting an undercutting of the SAL by the more moist marine mixed layer (Karyampudi and Carlson 1988; Karyampudi et al. 1999). Low humidity values are also seen at heights above the SAL over the eastern Atlantic and eastward to Saudi Arabia. A relative maximum in relative humidity occurs over both Saudi Arabia and western Africa at middle levels $(\sim 600-500 \mathrm{hPa})$ within the upper portion of the SAL. This midlevel moistening ${ }^{3}$ is the result of deep dry convective mixing that drives the boundary layer profiles of potential temperature and specific humidity to nearconstant values (Carlson and Prospero 1972; Messager et al. 2009). Taken within the context of the very low background relative humidity suggested by the humidity above the SAL, these results indicate that, on average, the processes that produce the SAL cause drying of the lower levels (primarily below the 700-600-hPa layer) and moistening of the middle levels (600 to as high as $400 \mathrm{hPa}$ ). The transition from dry to relatively moist conditions occurs on average in the $700-600-\mathrm{hPa}$ layer, although considerable variability likely exists in individual SAL events. Although not described in this context, Lidar Atmospheric Sensing Experiment (LASE), rawinsondes, and dropsonde

\footnotetext{
${ }^{3}$ By moistening, we do not imply an approach to saturation, but merely that the relative humidity is higher than it would likely be in the absence of the deep convective mixing given the ambient RHs in the nearby environment.
} 

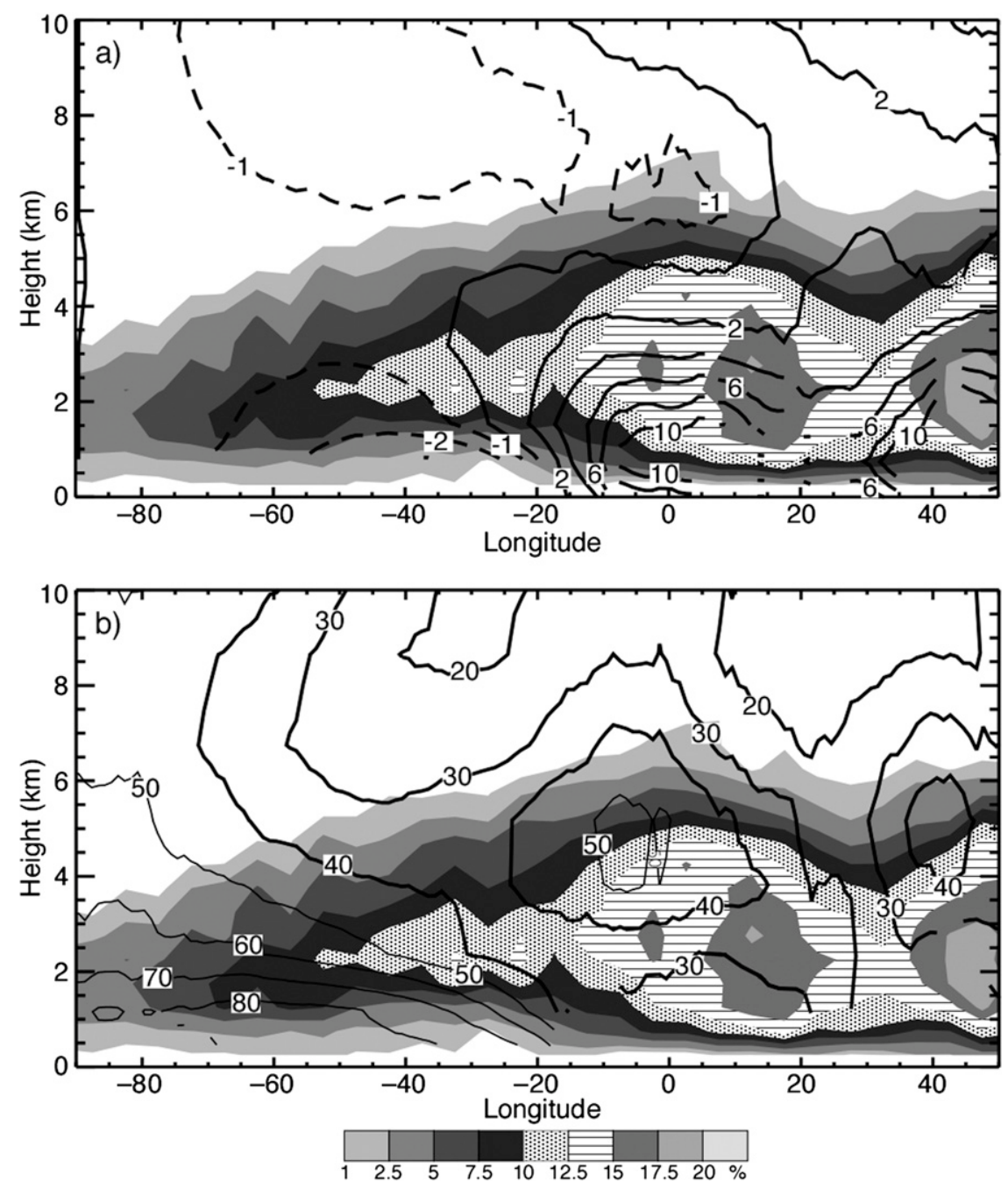

FIG. 2. JJA 2006-07 mean dust detection frequency (shading) from CALIPSO averaged between $12.5^{\circ}$ and $32.5^{\circ} \mathrm{N}$ and August 2003-08 (a) mean potential temperature perturbation (1$\mathrm{K}$ intervals for negative values, $2 \mathrm{~K}$ for positive values) and (b) $\mathrm{RH}(\leq 40 \%$, thick lines; $\geq 50$, thin lines) from AIRS-AMSU between $18^{\circ}$ and $30^{\circ} \mathrm{N}$. The temperature field is the perturbation from the profile at $-90^{\circ} \mathrm{W}$ (left edge of figure).

measurements obtained during the NASA African Monsoon Multidisciplinary Activities experiment in 2006 (Zipser et al. 2009; Ismail et al. 2010) suggest that this vertical structure of humidity is often found at the west coast and downstream of Africa over the eastern Atlantic as well.

\section{b. Dry air over the Atlantic Ocean and within the $S A L$}

In this section, we use the AIRS-AMSU data and GFS analyses to highlight climatological aspects of the SAL's moisture distribution and place it within the context of the large-scale environment over the Atlantic. Average conditions for August (2003-08) are highlighted. To demonstrate that this month is qualitatively similar to other months during the hurricane season in which the SAL is active, Fig. 3 shows the 700-600-hPa mean relative humidity averaged over 2003-08 for the individual months of June-September. Despite the greater activity of SAL outbreaks earlier in the hurricane season (Engelstaedter et al. 2006; Dunion and Marron 2008), the relative humidity distribution shows remarkable consistency through the period, with areas of minimum relative humidity located over the eastern portion of North Africa and also over the eastern Atlantic off of the northwestern African coast. As the North African continent heats up through August, relative humidities over western Africa increase rather than decrease, possibly as a result of deep dry convective mixing (Carlson and Prospero 1972) or increases in nearby 

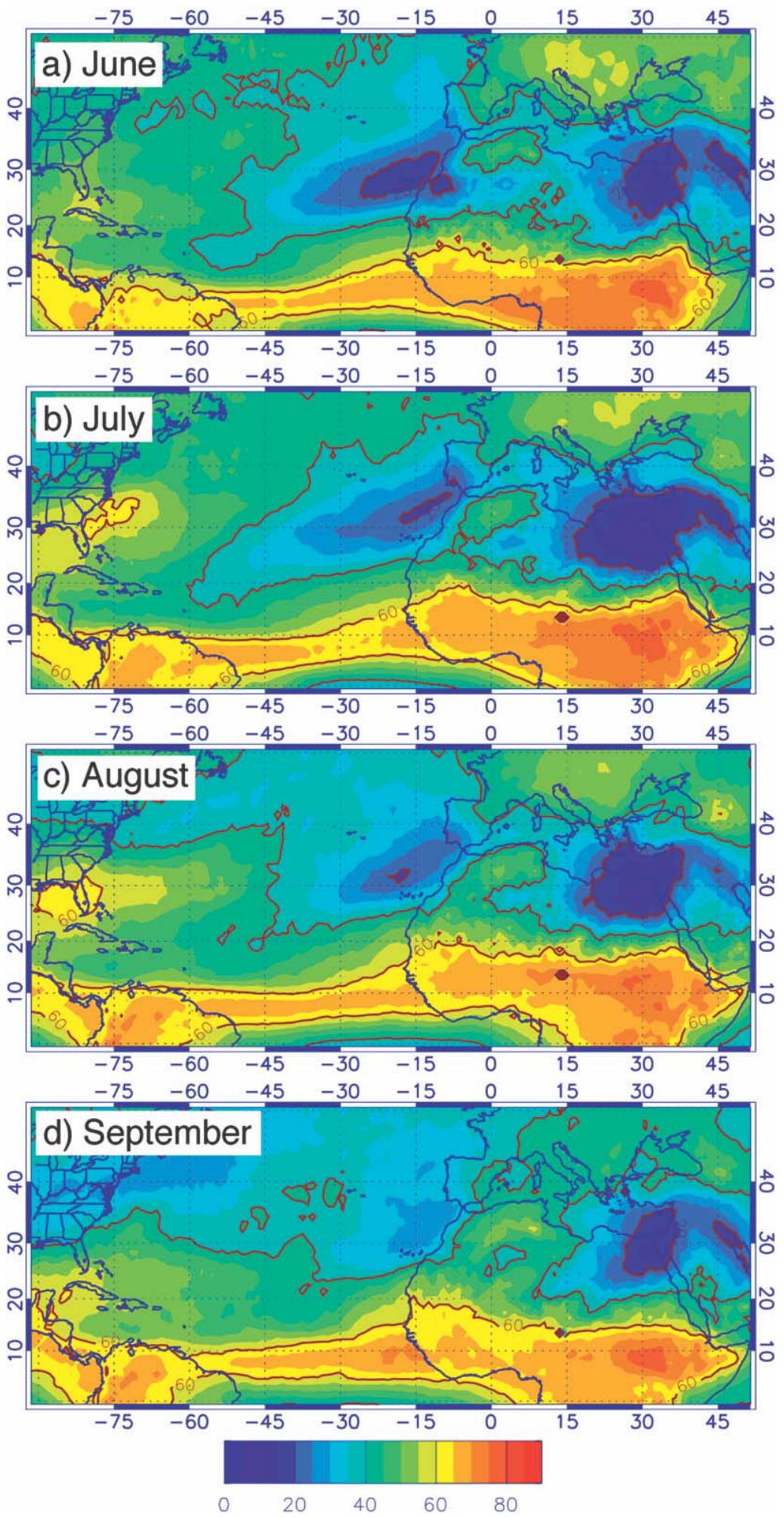

FIG. 3. Mean RH in the 700-600-hPa layer averaged over 2003-08 for (a) June, (b) July, (c) August, and (d) September from AIRS-AMSU. Red contours are drawn at $20 \%, 40 \%$, and $60 \% \mathrm{RH}$. 
precipitation associated with African easterly waves (Duvel 1990; Grist and Nicholson 2001; Grist 2002).

The ability of AIRS-AMSU to detect the hot Saharan air mass is demonstrated in Fig. 4, which shows the August 2003-08 temperatures and relative humidities at several levels. Very warm $700-$ and $850-\mathrm{hPa}$ temperatures (Figs. 4d and 4g) coincide with the mean dust layer (thick outlines in Fig. 4), with the warmest air over Africa and Saudi Arabia and with temperatures diminishing rapidly as the air moves westward. As seen in Fig. 1, precipitation is heaviest in the intertropical convergence zone (ITCZ) south of the warm SAL region and, like dust and the warm air, also diminishes substantially by $40^{\circ} \mathrm{W}$. A possible reason for this close relationship between dust/warm air and precipitation is that the frontogenetic properties of the warm SAL provide an indirect vertical circulation that enhances cloud development, as proposed by Karyampudi and Carlson (1988). If true, then the SAL would be expected to have a positive influence on cyclone development by fostering mean ascent to its south. ${ }^{4}$

The mean environment of the SAL can also be ascertained from Fig. 4. For example, the temperature (relative humidity and specific humidity) at $500 \mathrm{hPa}$ (500-400 hPa) in Fig. 4a (Figs. 4b and 4c) represent conditions at, or just above, the top of the SAL. Here, we find three primary dry regions unrelated to the SAL: one over the eastern Mediterranean and northern Egypt, another over the eastern Atlantic northwest of the West African coast, and the third over the central Atlantic Ocean. The dry area over the eastern Mediterranean region is collocated with an area of significant warming (Fig. 4a). The displacement of this warm area northwest of the area of low-level warming over the desert and just south of a region of strong mean subsidence (Fig. 5b) suggests that the warming and drying are subsidence driven. The dry region off of the northwest African coast also shows signs of warming in a region of large-scale subsidence. Relatively high humidity is found extending northward from the ITCZ over western Africa, a possible indication of the moistening near the top of the SAL (Carlson and Prospero 1972) and perhaps horizontal transport from the ITCZ (Ismail et al. 2010).

The relative humidity and specific humidity in the 700-600-hPa layer (Figs. 4e and 4f) are nearly identical to those at $500-400 \mathrm{hPa}$, but with somewhat higher humidity overall. Three characteristic moisture regimes are found in the eastern Atlantic: a moist zone in the

\footnotetext{
${ }^{4}$ This fact does not preclude the SAL from simultaneously having negative influences as well, particularly as a result of dry-air ingestion into developing mesoscale cyclonic systems.
}

tropical precipitation belt, a very dry region on the northern edge of the dust layer, and an intermediate humidity region within the dust layer. This zone of intermediate humidity within the SAL can be partly explained by westward transport of relatively moist midlevel SAL air. In addition, as suggested by Ismail et al. (2010) (see also Fig. 8c), southerly flow just above the AEJ and in association with AEWs may transport moister air from convective regions to the south.

At $700 \mathrm{hPa}$, the influence of the Sahara becomes readily apparent in the temperature field (Fig. 4d), with air temperatures over the Sahara that are 3-5 K greater than the air over nearby Atlantic waters. If one holds the vapor mixing ratio constant at a value representative of the air over the Sahara, then a temperature drop from $\sim 286 \mathrm{~K}$ by $3-5 \mathrm{~K}$ as the air moves westward at this level would account for a $9 \%-16 \%$ rise in relative humidity (from $\sim 41 \%$ to $57 \%$ ). The observed decrease over the Atlantic to the west, therefore, is opposite of our expectations from drying by Saharan-induced warming. It also implies that the relative humidity variability seen in Fig. 4e arises largely from variations in the vapor mixing ratio (as confirmed in Fig. 4f) and suggests that the observed drying toward the west is likely caused by some other mechanism such as subsidence.

In the 850-700-hPa layer (Fig. 4h), the zone of intermediate humidity over the western Sahara and the eastern Atlantic is absent, replaced by very dry air caused by the strong low-level warming over the Sahara. The 850-hPa temperatures (Fig. $4 \mathrm{~g}$ ) show values as much as 10-12 K warmer over the Sahara than air over nearby Atlantic waters. Again, holding vapor mixing ratios constant would suggest that a drop in temperature from $\sim 305$ to $293 \mathrm{~K}$ (Fig. 4g) would result in an increase in relative humidity from $\sim 27 \%$ to $56 \%$, approximately in line with the change seen in Fig. 4h. The decrease in relative humidity seen over northern Africa is therefore consistent with the expected drop caused by strong surface warming. Thus, the drying impact of the Sahara on the mean moisture field is seen primarily below the $700-600-\mathrm{hPa}$ layer over Africa in the AIRS data, but fades quickly as the air cools as it moves westward.

The relative humidity in the 700-600-hPa layer from the GFS analyses (Fig. 5a) is nearly identical to that from AIRS (Fig. 4e). The monthly mean wind vector pattern and vertical velocities (Fig. 5b, depicting only downward motions) show that the driest regions are located at the terminus of northerly descending airflow associated with midlatitude troughs on either side of a Saharan midlevel high pressure system. Over the Atlantic, the dry tongue that extends from northwest Africa to the central Atlantic is associated with descending flow on the northern side of the AEJ, suggesting that the jet or the SAL may partly 
a) $500 \mathrm{hPa}$ Temperature

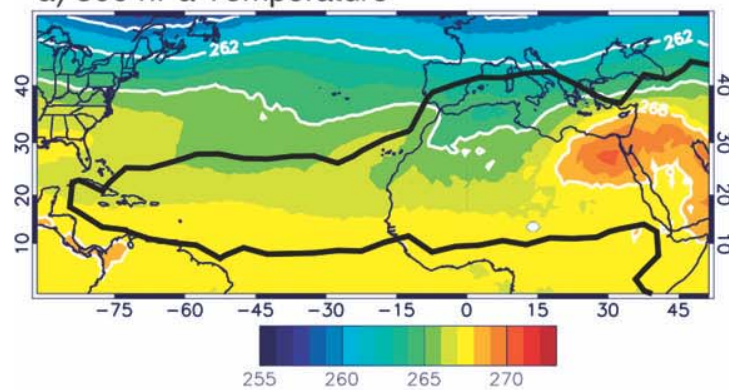

d) $700 \mathrm{hPa}$ Temperature

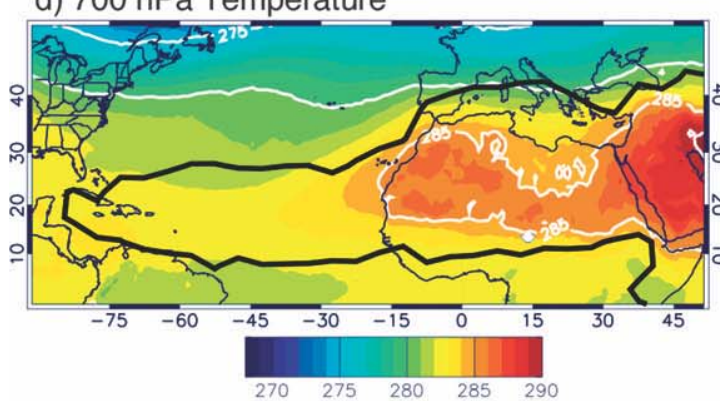

g) $850 \mathrm{hPa}$ Temperature

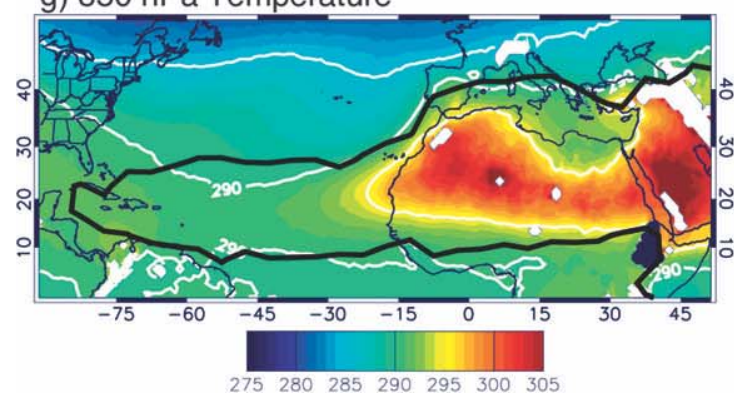

b) $500-400 \mathrm{hPa} \mathrm{RH}$

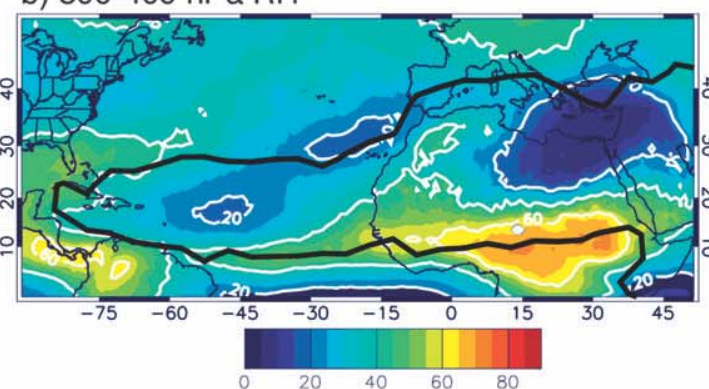

e) $700-600 \mathrm{hPa} \mathrm{RH}$

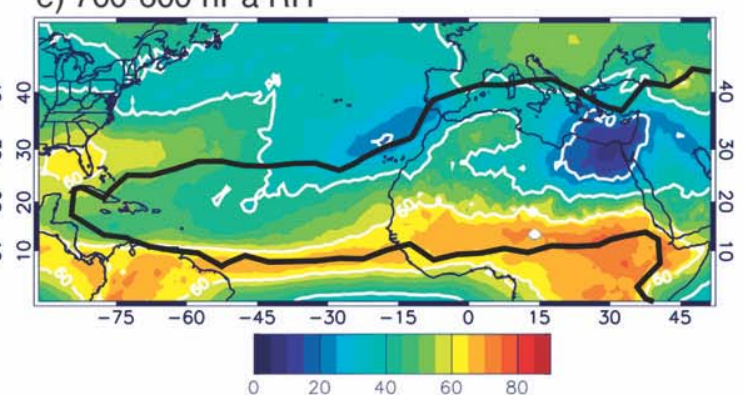

h) $850-700 \mathrm{hPa} \mathrm{RH}$

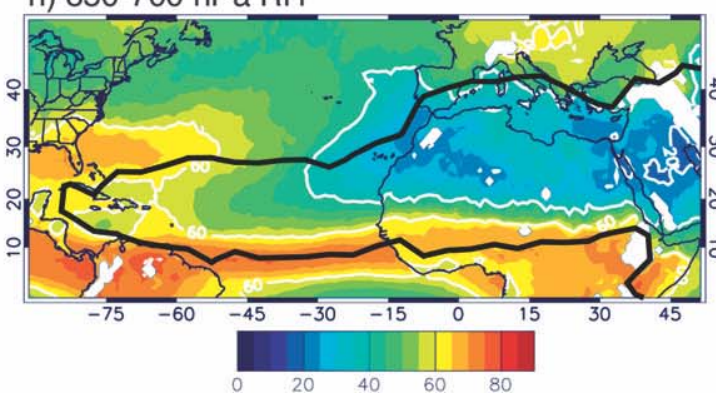

c) $500-400 \mathrm{hPa}$ qv

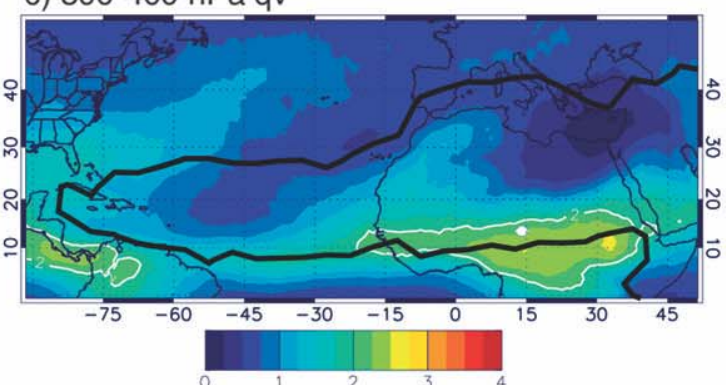

f) $700-600 \mathrm{hPa}$ qv

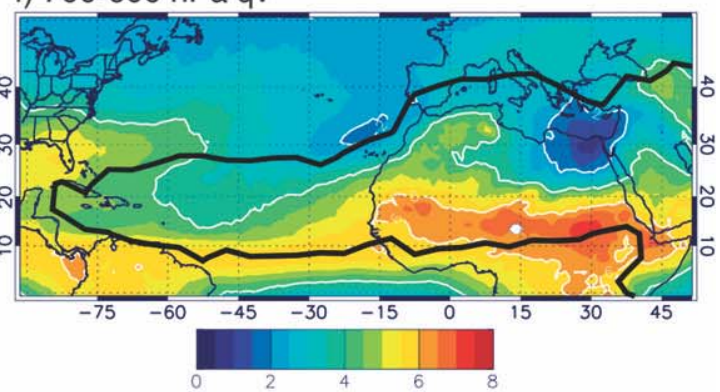

i) $850-700 \mathrm{hPa}$ qv

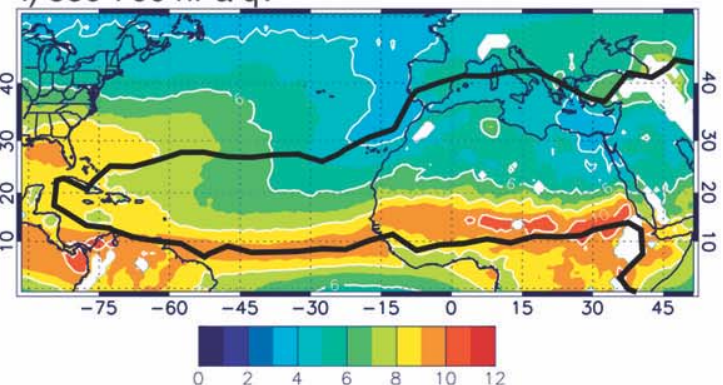

FIG. 4. Mean distributions for August 2003-08 of AIRS-AMSU-derived (a),(d),(g) temperature (in K) at 500, 700, and 850 hPa, respectively; and (b),(e),(h) RH (\%); and (c),(f),(i) specific humidity $\left(\mathrm{g} \mathrm{kg}^{-1}\right)$ for the 500-400-, 700-600-, and 850-700-hPa layers, respectively. The thick black line in each panel is the $20 \%$ frequency contour for the dust layer from Fig. 1 . 

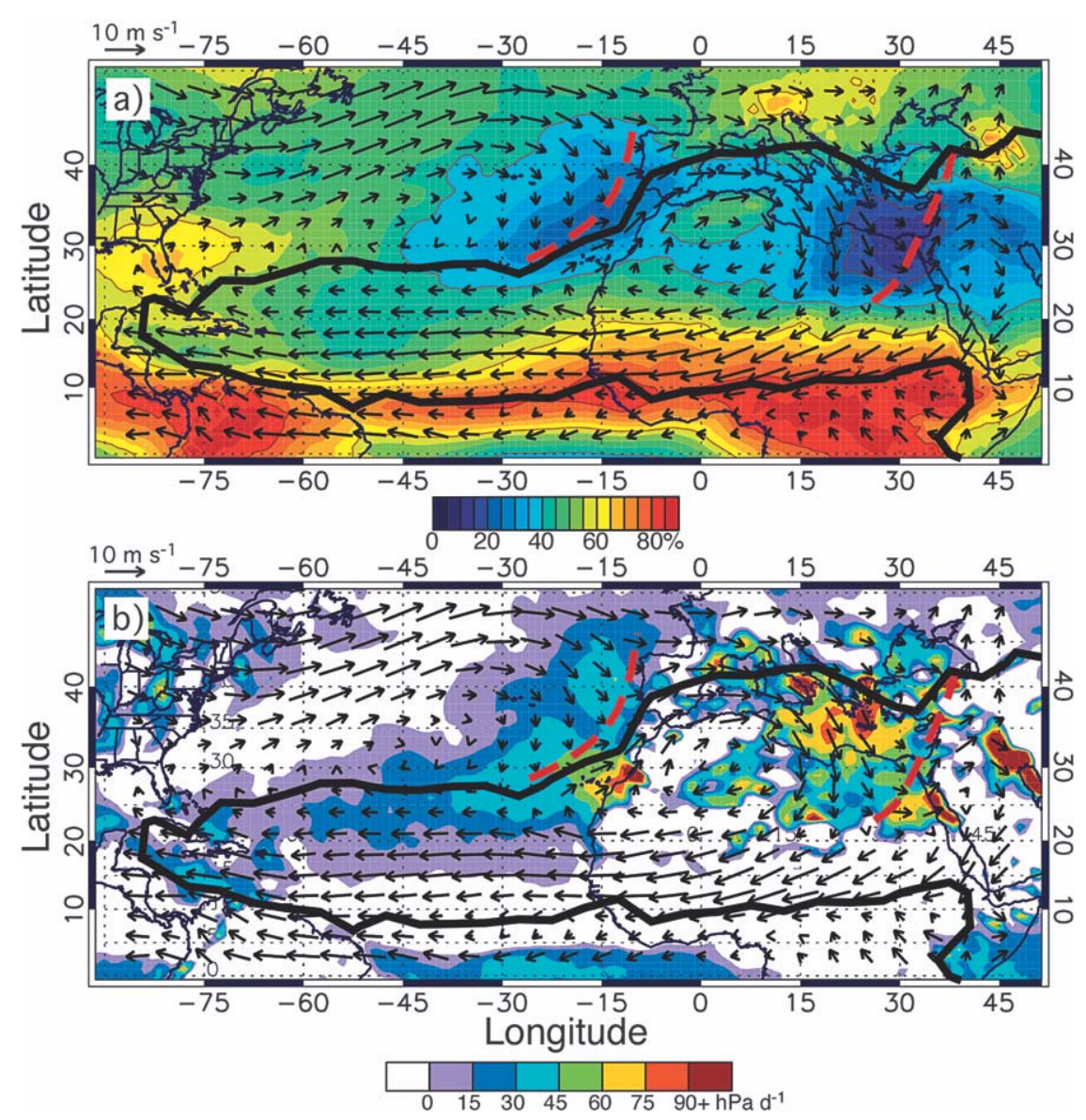

FIG. 5. August 2003-08 mean GFS-derived 700-600-hPa layer (a) RH and (b) vertical velocity (in $\mathrm{hPa}$ day ${ }^{-1}$ ) over the Atlantic and northern Africa. Arrows show the 700-hPa mean flow vectors. The thick black outline is the $20 \%$ frequency contour for the dust layer from Fig. 1. Red dashed lines indicate trough axes.

contribute to this descending flow. Results presented are typical of other months during the hurricane season.

We can further expand our view to look at the global distribution of monthly averaged (again, August 2003$08)$ relative humidity and vertical motions from GFS fields (Fig. 6). Similar to the Atlantic, regions of dry air can be found in the eastern portions of major ocean basins in both the Northern and Southern Hemispheres. For example, over the northeastern Pacific Ocean, dry air occurs from the coast of California toward the southwest into the tropical Pacific. Monthly averaged wind vectors (Fig. 6a) reveal that these dry regions are the product of semipermanent pressure systems, including the Pacific and Bermuda highs, a Saharan high, a monsoonal low over northern India, and subtropical high pressure regions in the Southern Hemisphere. The dry oceanic areas are zones of large-scale subsidence on the eastern and equatorward sides of the high pressure systems (Fig. 6b). The role of subsidence is well known
(Riehl et al. 1951; Picon and Desbois 1990; Sherwood 1996; Soden and Bretherton 1996; Pierrehumbert 1998; Soden 1998; Zhang and Pennington 2004), but has been largely absent in discussions of the SAL (e.g., DV; Jones et al. 2007; Wu 2007; Dunion and Marron 2008). Figure 6 should not be interpreted as saying that midlatitude dry air moving around the oceanic high pressure systems is the main source of dry tropical air. While midlatitude dry air frequently intrudes into the tropics and moves westward, a large fraction of the dry midlevel air in the tropics is likely derived from subsiding tropical air equatorward of the highs. These results suggest that large-scale processes dominate the distribution of moisture over the oceans, including the Atlantic. They also imply that the Sahara is likely a smaller modulating influence, not the main determinant, of the tropical Atlantic moisture distribution since its drying effects, on average, are generally confined to levels below $\sim 700-600 \mathrm{hPa}$ and rapidly diminish to the west as the air cools. 

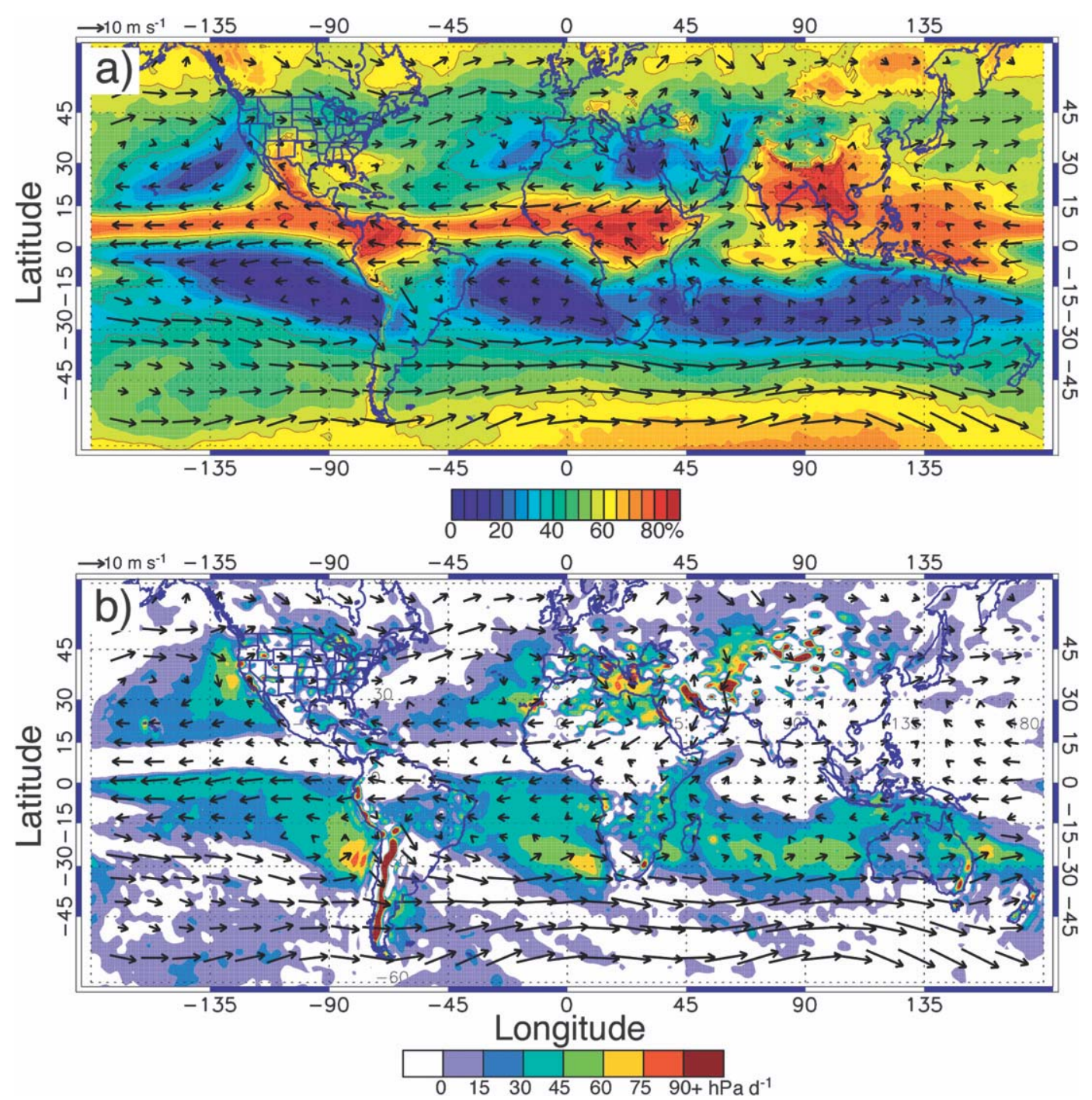

FIG. 6. August 2003-08 mean GFS-derived 700-600-hPa layer (a) RH and (b) vertical velocity (in hPa day ${ }^{-1}$ ) over the globe. Arrows show the 700-hPa mean flow vectors.

Not only does the AEJ mark the region of strong temperature gradient on the southern side of the SAL (Karyampudi and Carlson 1988), it also lies within the zone of strong meridional moisture gradient over the Atlantic and Africa (Fig. 5a). Because the AEJ is a source of energy for waves, easterly waves readily form and move westward across the tropics. These waves, lying in this zone of strong moisture gradient, then produce perturbations in the moisture field with dry (moist) air westward (eastward) of trough axes. The implication of these results is that the dry SAL air is, to first order, the dry sector of an easterly wave. This dryness is enhanced below the 700-600-hPa layer by surface heating over the Sahara, but this enhancement diminishes as the air cools while moving westward.

\section{Vertical shear and increased stability}

Studies by Frank (1970), Burpee (1972), Landsea and Gray (1992), Thorncroft and Hodges (2001), and Ross and Krishnamurti (2007), among others, have demonstrated that the AEJ plays an instrumental role in the formation of tropical cyclones over the Atlantic, with most storms developing to the south of the AEJ axis. The southern side of the jet is characterized by strong cyclonic vorticity, thereby providing a vorticity-rich environment for cyclogenesis. The AEJ is also an energy source for AEWs, which grow by baroclinic and barotropic instability (Burpee 1972; Thorncroft and Hoskins 1994a,b). Cumulus convection south of the AEJ, particularly in the ITCZ, contributes to wave growth by 
producing reversals in the meridional gradient of potential vorticity (Mass 1979; Hsieh and Cook 2005). DV suggested that the AEJ increases the low- to midlevel easterlies, thereby increasing the deep-layer vertical wind shear in a way that could negatively impact developing cyclones. They show vertical shear estimates for the cases of Hurricanes Isaac and Joyce (see their Fig. 6), with peak shear associated with the SAL northeast of Joyce of about $35 \mathrm{~m} \mathrm{~s}^{-1}$ between the 150-350- and 700-925-hPa layers. Although the method of estimating shear from the satellite winds and complications associated with the removal of the vortex cause significant uncertainty in the shear estimate, DV's Fig. 6 suggests that the shear was primarily on the periphery of the storm. Whether and by which means storms are detrimentally impacted by vertical shear on their periphery has not been well established. In most previous studies of vertical wind shear impacts on tropical cyclones, the "detrimental" shear was thought to be that existing over the core (the center and out to some specified radius) of the storm (Marks et al. 1992; Franklin et al. 1993; Reasor et al. 2000; Black et al. 2002; Corbosiero and Molinari 2002, 2003; Rogers et al. 2003; Chan et al. 2004; Braun et al. 2006; Braun and $\mathrm{Wu}$ 2007; Chen et al. 2006) and was often assumed to be horizontally uniform in modeling studies (Jones 1995; Frank and Ritchie 1999, 2001; Wong and Chan 2004). For this study, we assume that the presence of an AEJ near the periphery of a storm is not necessarily detrimental to storm development. This assumption is supported by the composite results in section 5 in which it is shown that, in fact, an adjacent AEJ is characteristic of most Atlantic storms.

Analysis of GFS fields for the tropical cyclone events contained in the composite fields discussed in the next section (between 2001 and 2008) suggests that the AEJ generally provides a key source of vorticity and frequently forms the northern portion of the storms. Hurricanes Florence and Helene in 2006 are provided here as typical examples (Fig. 7). On 3 September (Fig. 7a), a broad jet is apparent at $700 \mathrm{hPa}$, extending from the western coast of Africa to $\sim 50^{\circ} \mathrm{W}$ at a latitude of $\sim 17^{\circ}$ $18^{\circ} \mathrm{N}$. A region of enhanced cyclonic vorticity (not shown) that would develop within $6 \mathrm{~h}$ into a tropical depression and later into Hurricane Florence is located south of the jet between $40^{\circ}-50^{\circ} \mathrm{W}$ and $12^{\circ} \mathrm{N}$. The system becomes Tropical Storm Florence on 5 September (Fig. 7c) with the remnants of the AEJ now composing the northern part of the storm circulation. Warm temperatures associated with the SAL (Fig. 7b) have cooled by several degrees by 5 September (Fig. 7d). Eight days later (Fig. 7e), as Florence moves northeastward off the U.S. east coast, a new wave has emerged off of the western African coast to form a tropical depression in association with a strong AEJ and a pocket of warm SAL air (Fig. 7f). As with Florence, the cyclonic vortex develops south of the jet. Over the next several days (Fig. 7g), Tropical Storm and later Hurricane Helene forms, the trailing (eastern) portion of the jet becomes the northern part of the storm circulation, and the region of warm SAL air cools by $2-4 \mathrm{~K}$ (Fig. 7h). Cooling rates are approximately $1.5^{\circ}-2^{\circ} \mathrm{C}$ day $^{-1}$, consistent with, but slightly larger than, the estimates of Carlson and Prospero (1972) of about $1.6 \mathrm{~K} \mathrm{day}^{-1}$.

The relationship between the dust layer, precipitation, the AEJ, and the large-scale meridional circulation is demonstrated in Fig. 8 for 2 September 2006, immediately before the formation of Florence. Strong easterly winds at $700 \mathrm{hPa}$ extend from the African coast to $\sim 50^{\circ} \mathrm{W}$ with peak winds along or near the southern and leading edges of the dust layer (Fig. 8a). The heaviest precipitation is located south of the leading portion of the dust outbreak. Meridional cross sections formed by averaging between $20^{\circ}$ and $40^{\circ} \mathrm{W}$ show the AEJ centered near $16^{\circ}-17^{\circ} \mathrm{N}$ and $\sim 650 \mathrm{hPa}$ (Fig. $8 \mathrm{~b}$ ). The vertical circulation exhibits low-level convergence and strong ascent in the ITCZ on the southern side of the AEJ and sinking motion to the north of the jet. Although there is weak rising motion beneath the jet, deep saturated ascent (Fig. 8c) is confined to the region to the south where vertical shear associated with the AEJ is weak. Thus, the deep convection is confined to the cyclonic vorticityrich region south of the jet, enabling development of the tropical cyclone. Since the jet does not move over the region of deep convection (Fig. 7), the vertical shear associated with the AEJ, even if it suppresses deep convection under it, does not generally inhibit development. The region of dust extends meridionally from about $13^{\circ}$ to $27^{\circ} \mathrm{N}$ (see box in Fig. 8c), with low-level drying in the 900-700-hPa layer and moistening in the 700-500-hPa layer. Substantial dry air resides above and just north of the dust layer, with the streamlines indicating strong subsidence through the depth of the free troposphere in the dry layer. The examples of Hurricanes Florence and Helene are typical of hurricanes developing from AEWs in the central and eastern Atlantic based upon GFS analyses and satellite data for 2001-08.

The warm SAL air is found to the north of the AEJ (Karyampudi and Carlson 1988), with the developing storms typically located south of the jet very close to the southern edge of the warm layer (see Fig. 7), in other words, near the zone of strong meridional temperature gradient on the southern side of the SAL. To the extent that the airflow is in thermal wind balance (Cook 1999), the fact that the jet usually forms the northern side of developing storms implies that the warmer air of the SAL, and hence the greater thermodynamic stability 

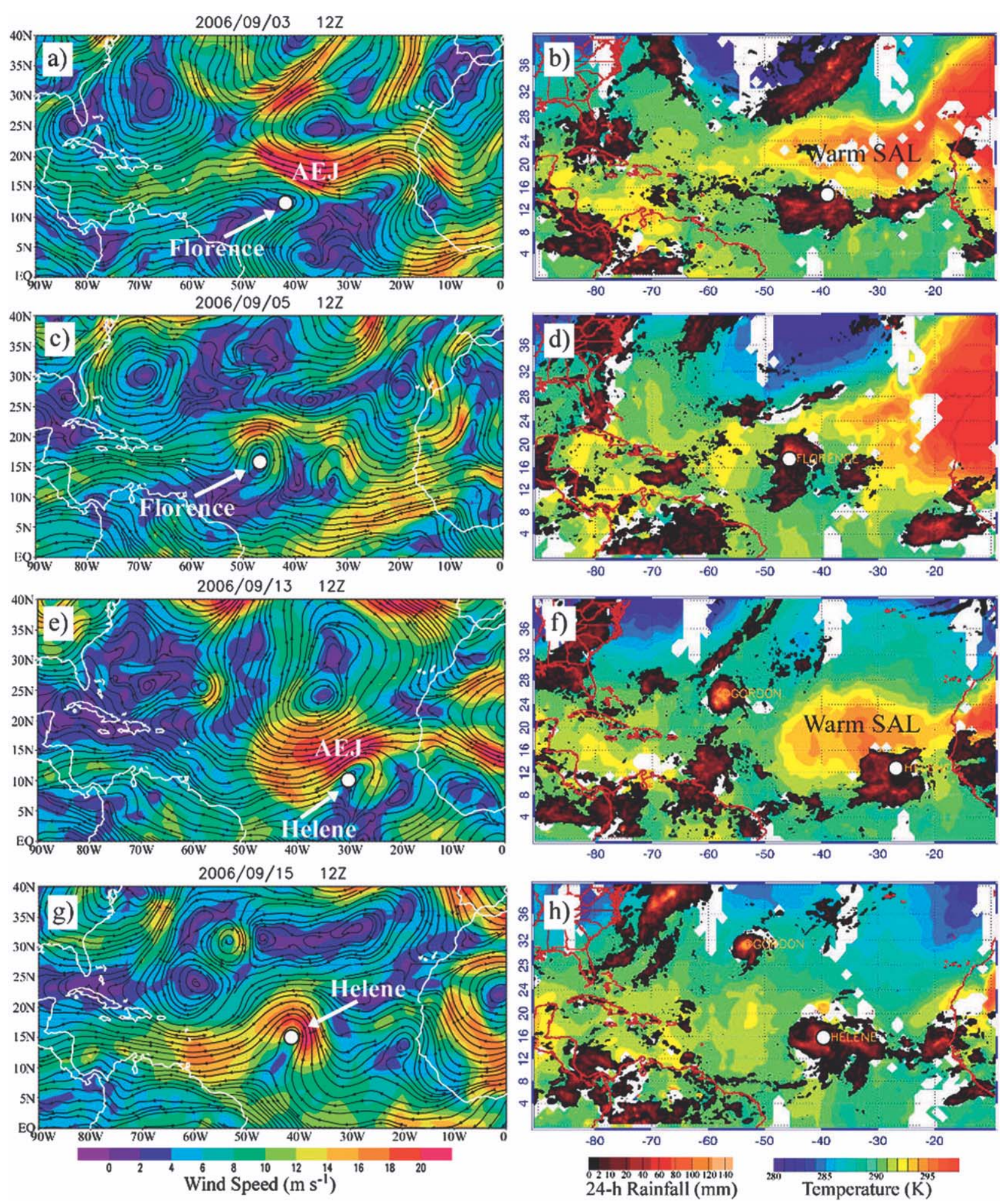

FIG. 7. (left) GFS-analyzed isotachs and streamlines at $700 \mathrm{hPa}$ for the indicated days and times. Plots show the evolution of the easterly jet for Hurricanes Florence and Helene (indicated by white dots) during September 2006. (right) The corresponding AIRS-AMSU 850-hPa temperature and TRMM 24-h accumulated rainfall. 

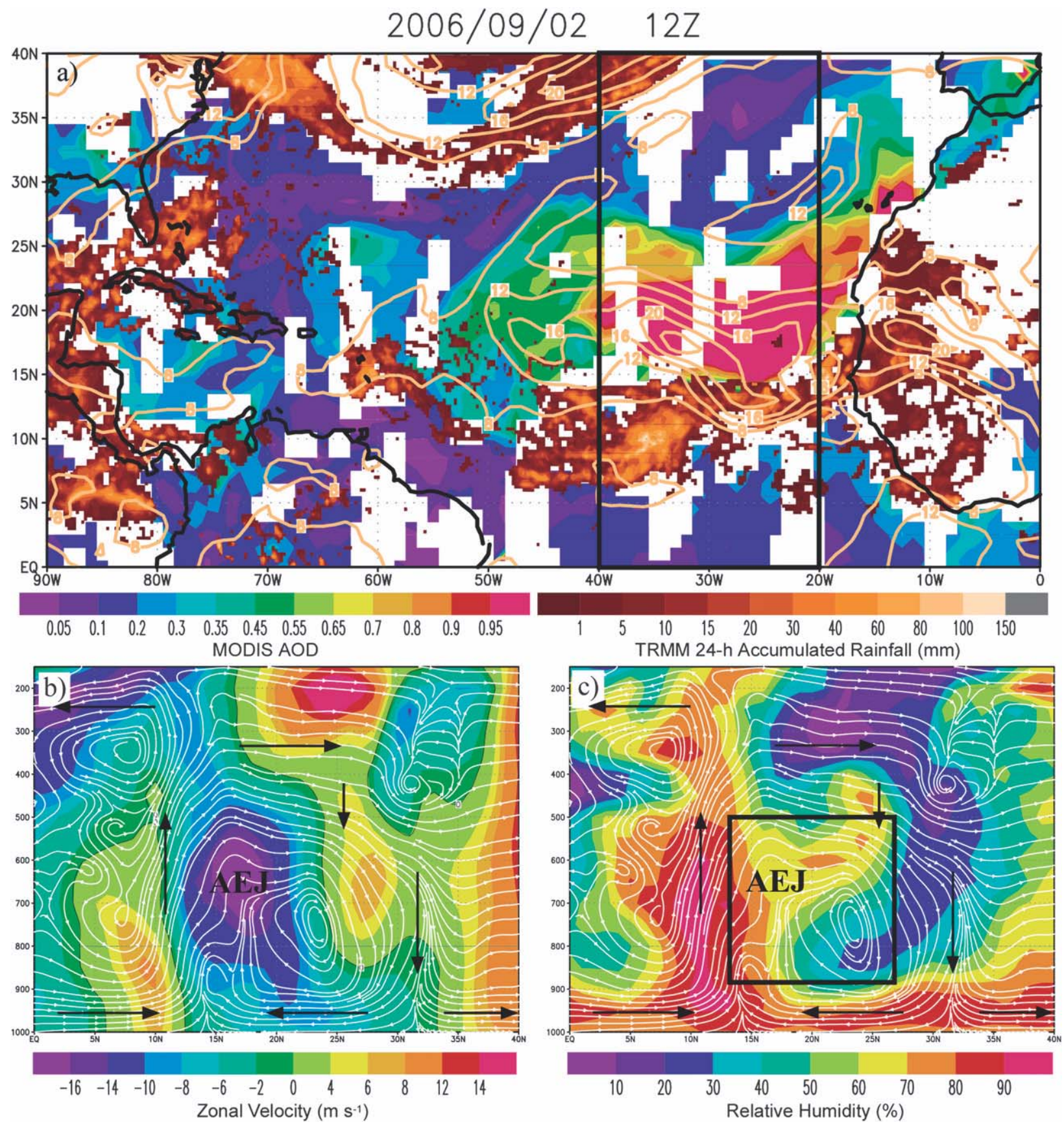

FIG. 8. (a) MODIS AOD, TRMM 24-h accumulated rainfall, and GFS 700-hPa winds (isotachs, contours at $4 \mathrm{~m} \mathrm{~s}^{-1}$ intervals starting at $8 \mathrm{~m} \mathrm{~s}^{-1}$ ) for 1200 UTC 2 Sep 2006. Vertical cross sections of analyzed meridional circulation (streamlines) and (b) zonal wind and (c) RH averaged between $20^{\circ}$ and $40^{\circ} \mathrm{W}$ as outlined by the black box in (a). The location of the AEJ is indicated, while arrows highlight the direction of the mean circulation. The black box in (c) indicates the meridional extent of the SAL suggested by the MODIS data in (a) and approximate base and top heights for the SAL.

caused by it overriding a cooler marine layer, are usually confined to areas north of developing storms (see, e.g., the right column in Fig. 7). As a result, this higherstability air would not be expected to often impact precipitating regions of the storms. In fact, it may help to focus convection along the southern border of the SAL and the cyclonic side of the AEJ, which would aid in the development of tropical disturbances. 


\section{A composite view of the SAL and hurricanes}

The case studies above are only two examples of many that can be shown. To demonstrate the generality of the results and to explore some of the differences between intensifying and weakening storms, a composite of the evolution of storms developing from easterly waves (and in association with SAL outbreaks) is examined. See section 2 for a description of the compositing technique and Table 2 for a list of all storms included in the analysis. The composites over all storms of temperature at $850 \mathrm{hPa}$, wind speed at $700 \mathrm{hPa}$, and vertical motion and relative humidity in the 700-600-hPa layer are shown in Fig. 9 for times $t_{0-2}, t_{0}$, and $t_{0+2}$, where $t_{0}$ is the time the disturbance was first identified as a tropical depression or storm in the HURDAT dataset, hereafter termed the time of genesis. Two days prior to genesis (Fig. 9a), a broad AEJ is seen about $4^{\circ}$ north of the disturbance center (at the origin) and stretching from about $20^{\circ}$ to the west to $30^{\circ}$ or more to the east, with the maximum winds located just northeast of the disturbance center. A strong temperature gradient is present in the region of the jet associated with the SAL. The relative humidity field (Fig. 9d) shows the broad moist zone associated with the ITCZ, with dry air both to the north and south. North of the storm, the axis of the driest air lies northward of the axis of warmest air and typically lies along or near the northern border of the dust region (not shown), suggesting that the dryness is not solely, or even predominantly, the result of low-level warming and deep mixing over the Sahara. Strong upward motion (Fig. 9d) is collocated with the developing disturbance, with weaker upward motion elsewhere along the ITCZ. Subsidence is present over a broad region south of the ITCZ and in regions to the north, generally strongest near the axis of driest air. On the day of genesis (Figs. 9b and 9e), the jet is still present, but with a more localized wind maximum on the north side of the storm. The temperature gradient is weaker as the SAL air cools. The axis of driest air lies well north of the storm, coincident with the strongest subsidence. By 2 days after genesis (Figs. 9c,f), the jet is virtually gone, but with strong winds remaining on the north side of the storm, similar to the examples shown in Fig. 7. There is no longer a significant horizontal temperature gradient near the storm as the warmest SAL air has been left well to the east. Dry midlevel air is seen to begin wrapping around the western side of the storm and is also collocated with a broad region of subsidence that extends from the eastern side around to the western side of the storm. The composite results support the inferences from the examination of the individual cases above that the jet, at least as depicted by the NCEP GFS analyses, is less likely a source of vertical shear over the storms than it is a source of cyclonic vertical vorticity that convection south of the jet is able to concentrate into the core of the storm.

The composite results clearly demonstrate that the warm SAL air inhibits convection within the SAL (Fig. 10). Two days prior to genesis (Fig. 10a), strong $\mathrm{CIN}^{5}$ is seen within the region of the SAL, with a meridionally narrow, but zonally extensive, region of weak CAPE in the ITCZ. Convection, as inferred from the analyzed convective cloud cover, is restricted to the southern part of the weak-CAPE tongue that is outside of the region of high CIN. The region of high CIN is generally characterized by easterly to northeasterly low-level flow, while the region to the south is dominated by southeasterlies. These two flow patterns converge along the ITCZ and lead to a region of concentrated low-level vorticity within the area of highest convective cloud cover. Over the next 4 days (Figs. 10b and 10c), the convection moves along the southern boundary of the high-CIN region, gradually moving toward the leading nose of the SAL and toward a region of higher CAPE, all the while further concentrating the low-level vorticity within the convective-cloud-cover maximum. While the SAL is clearly suppressing convection north of the developing storm, it is not clear from the composite whether it inhibits the development of the storm in any way.

To address whether the SAL is having a negative impact, the storms in the composite were broken down into three categories: 1) those strengthening by $20 \mathrm{kt}$ or more between days 2 and 4 after genesis, 2) those weakening by any amount during the same time, and 3) all remaining storms. This breakdown yielded 18 storms in category 1 , 13 storms in category 2, and 10 storms in category 3 (Table 2). Composites were then constructed for categories 1 and 2. The following analysis focuses on characteristics of the SAL and environment, with particular emphasis on statistically significant differences between the composites for strengthening and weakening storms. The expectation is that differences between the two composites might provide some understanding of why some storms intensify and others weaken and the possible role of the SAL.

Figure 11 shows the CAPE and CIN for weakening storms (left panels) and strengthening storms (right panels). The left panels in Fig. 11 also show the 95\% and $99 \%$ confidence levels from the Student's $t$ test while the right panels show the correlation between the intensity change (between days 2 and 4 ) and either CAPE or CIN.

\footnotetext{
${ }^{5}$ The CAPE and CIN values are provided within the GFS analyses as both surface-based and layer-based values. Here, the surface-based values are shown, but there is little difference from the layer-based values in the composite.
} 

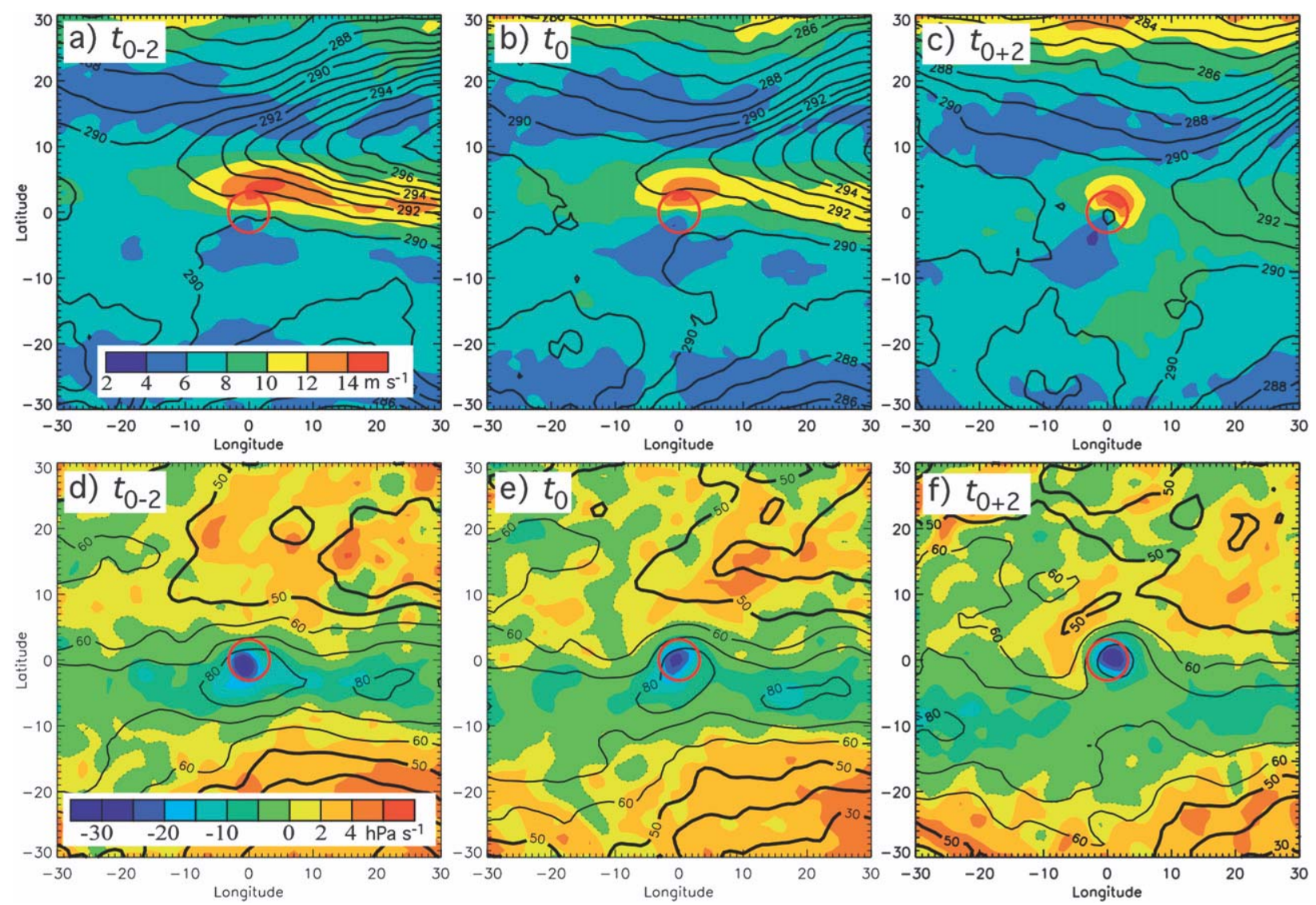

FIG. 9. GFS-derived fields for the full composite over all storms. (top) The horizontal wind speed (shading) at $700 \mathrm{hPa}$ overlaid with contours of 850-hPa temperature at $1-\mathrm{K}$ intervals for (a) $t_{0-2}$, (b) $t_{0}$, and (c) $t_{0+2}$. (bottom) The vertical motion (shading, $5 \mathrm{hPa} \mathrm{s}{ }^{-1}$ intervals for $\omega<0$ and $2 \mathrm{hPa} \mathrm{s}^{-1}$ for $\omega>0$ ) and RH (contours, $10 \%$ intervals; $\leq 50 \%$, thick lines; $\geq 60 \%$, thin lines) averaged over the 700-600-hPa layer. The red circle in each panel shows a $3^{\circ}$-radius circle centered on the storm.

For both cases, the patterns of CAPE and CIN are similar at $t_{0}$, as seen by the lack of significance or correlation. Strong CIN is present within the SAL just north of the convection in both weakening and strengthening cases, with relatively little statistically significant distinction between the two cases. Surprisingly, CAPE is somewhat higher in the weakening cases, but generally not significantly so. Similar results are obtained for other times.

An examination of 700-hPa zonal wind speeds at days $t_{0}$ and $t_{0+2}$ (Fig. 12) reveals few major differences other than the greater strength of the vortex in the strengthening cases at $t_{0}$. On the day of genesis, the AEJ is actually stronger in the strengthening cases, including well to the east of the vortex, than in the weakening cases (Figs. 12a and 12b), although the difference is statistically significant only in the vortex. By 2 days after genesis (Figs. 12c and 12d), the AEJ has weakened in the strengthening cases, but has remained about the same in the weakening cases. On this day, the jet is significantly stronger in the weakening cases along portions of the AEJ east of the vortex, but by days 3 and 4 (not shown) the differences are no longer significant. The weak degree of significance in the region of the AEJ only on day 2 and only east of the vortex suggests that vertical shear associated with enhanced easterlies associated with the AEJ is, at best, only a weak negative influence on tropical storm development. The stronger AEJ in the weakening cases during day $t_{0+2}$ may also be a result of the lack of development of the storm, which might otherwise grow at the expense of the energy of the jet.

Differences in the relative humidity and meridional velocities at $700 \mathrm{hPa}$ become significant only by day 2 (Fig. 13). The broad-scale relative humidity structure is quite similar between the two cases, with the main areas of significant differences found on the western and eastern sides of the vortex where the humidity is $\sim 10 \%-$ $20 \%$ lower in the weakening cases. The drier air to the east is found at the leading edge of the enhanced easterlies associated with the AEJ in the weakening cases, 

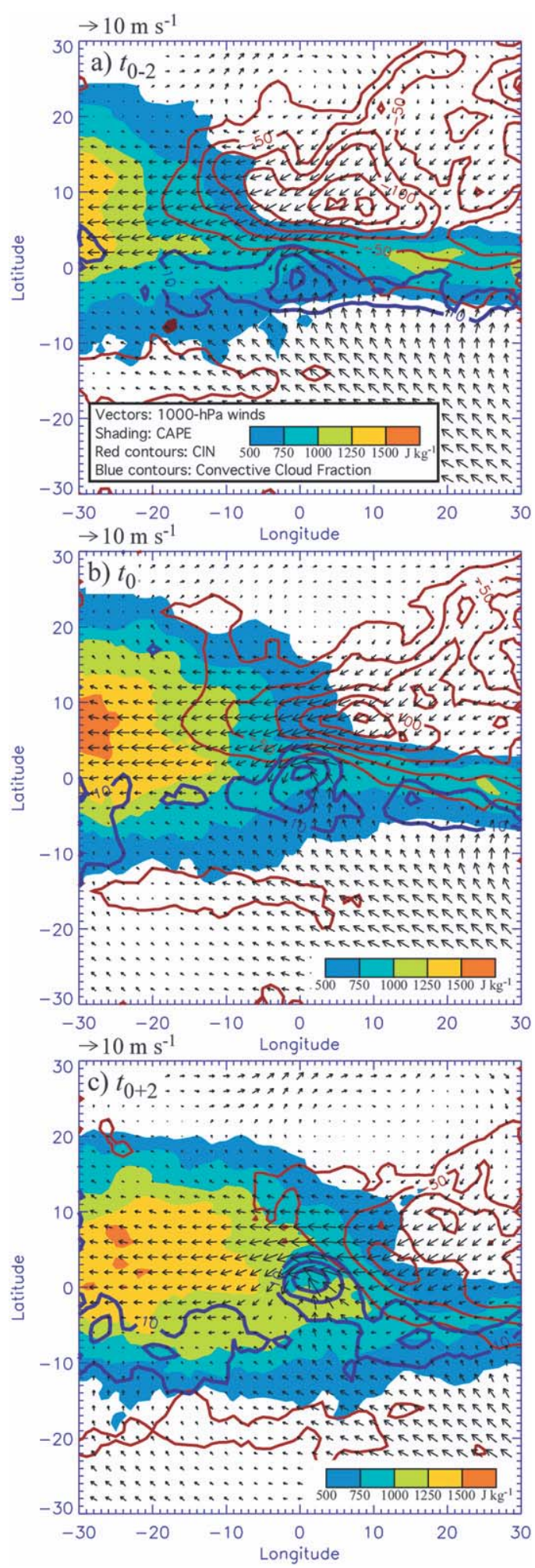

FIG. 10. GFS-derived fields for the full composite over all storms. Fields shown are CAPE (shaded), CIN (red contours, $25 \mathrm{~J} \mathrm{~kg}^{-1}$ intervals), convective cloud coverage (blue contours, $5 \%$ intervals starting at $10 \%$ ), and $1000-\mathrm{hPa}$ vector winds for days (a) $t_{0-2}$, (b) $t_{0}$, and (c) $t_{0+2}$. but also at the leading edge of the stronger and wider region of southerlies on the eastern side of the vortex in the strengthening cases. This pattern can be explained in two different ways. First, the lower humidity in the weakening cases may result from penetration of dry SAL air into the eastern sector of the vortex. Second, the meridional flow structures show a significantly stronger and wider vortex in the strengthening cases, which would advect greater moisture northward into that same sector. A similar pattern is present at $850 \mathrm{hPa}$ (not shown), with a somewhat broader area of statistical significance and stronger correlation.

At higher levels (600 and $400 \mathrm{hPa}$ in Fig. 14), the humidity patterns continue to show remarkable similarities and only small regions of significant differences. Dry air surrounds the northern semicircle around both the weakening and strengthening cases, but with lower humidity on the eastern side of the vortex in the weaker storms, similar to lower levels. Note, though, that this enhanced dryness is present even at levels above the SAL (Figs. 14c and 14d), suggesting that it is not necessarily caused by processes associated with the Sahara, but may instead be related more to the original origins of the air mass.

Some of the most pronounced differences between the weakening and strengthening cases occur at upper levels. At $400 \mathrm{hPa}$ on the day of genesis (Figs. 15a and $15 \mathrm{~b}$ ), a broad area of statistically different zonal winds and negative correlation is seen on the northern side of the vortex, primarily indicating the greater strength of the vortex and stronger easterlies (and weaker implied vertical wind shear) east of the storm in the strengthening cases. By 2 days after genesis (Figs. 15c and 15d), significantly stronger easterlies continue on the northern side of the vortex. Northwest of the storms, the weakening cases are associated with weak easterlies while the stronger storms are associated with westerlies that wind vectors indicate are partly a result of a much stronger anticyclone north of the strengthening cases.

Figure 16 shows the zonal wind speeds and vector winds at $200 \mathrm{hPa}$. On the day of genesis (Figs. 16a and 16b), the primary differences are found just north and northwest of the storm, with weakening storms characterized by a broad trough to the north or northeast of the storm and much stronger westerlies extending to near the storm center, while strengthening storms are located south of a ridge with westerlies displaced farther northward. Outflow from the strengthening storms is stronger and broader, which may reflect the more vigorous convection in those cases or a favorable interaction with the trough located poleward and to the west of the storm (Holland and Merrill 1984; Molinari and Vollaro 1989; Rappin et al. 2010). By day 2 (Figs. 16c and 16d), weak westerlies 

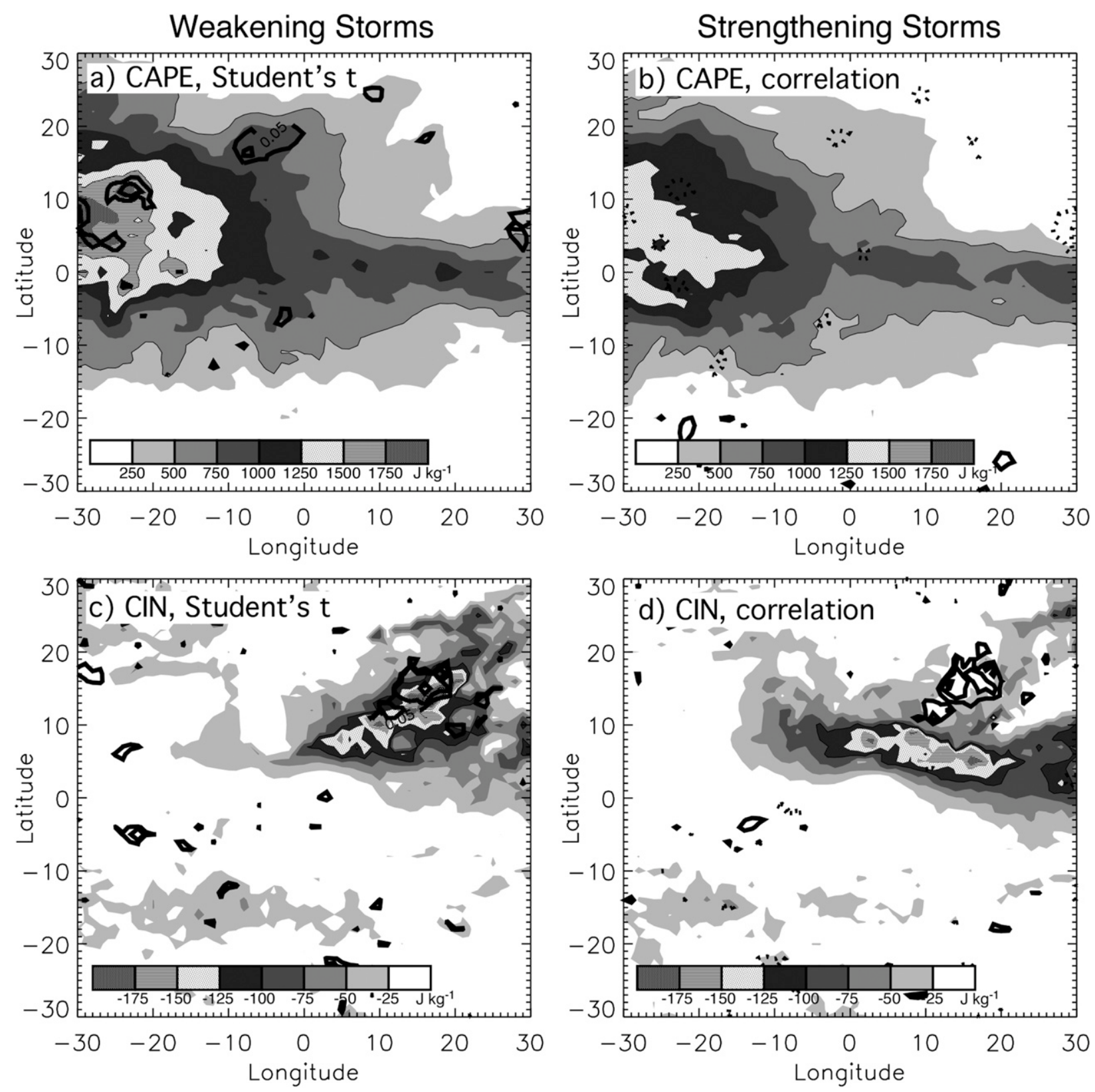

FIG. 11. GFS-derived fields for the composites over (left) weakening and (right) strengthening storms at time $t_{0}$. Fields shown by shading are (a),(b) CAPE and (c),(d) CIN. Contours in the left panels show locations where differences between the two composites are significant at the $95 \%$ and $99 \%$ confidence levels from the Student's $t$ test. Contours in the right panels show the correlation between the intensity change between days 2 and 4 and the fields being plotted, in this case, CAPE and CIN. Solid (dotted) contours show correlations $\geq 0.3$ $(\leq-0.3)$ at 0.1 intervals.

remain above the weakening storms and outflow is highly constrained. In the strengthening cases, a well-organized and broad anticyclonic outflow region has been established. Vertical wind shear (Fig. 17) between the 925-700and 300-100-hPa layers (similar to layers used in Fig. 6 of DV; note however that Fig. 17 still includes the effects of the vortex) shows strong shear over the weakening storms and much weaker shear in the strengthening cases. That the low- to midlevel easterlies are about the same in the two cases suggests that the increased deep-layer shear in the weakening cases is primarily a result of the stronger upper-level westerlies.

The results in this section suggest that the SAL is not a major determinant of intensity change in the several 

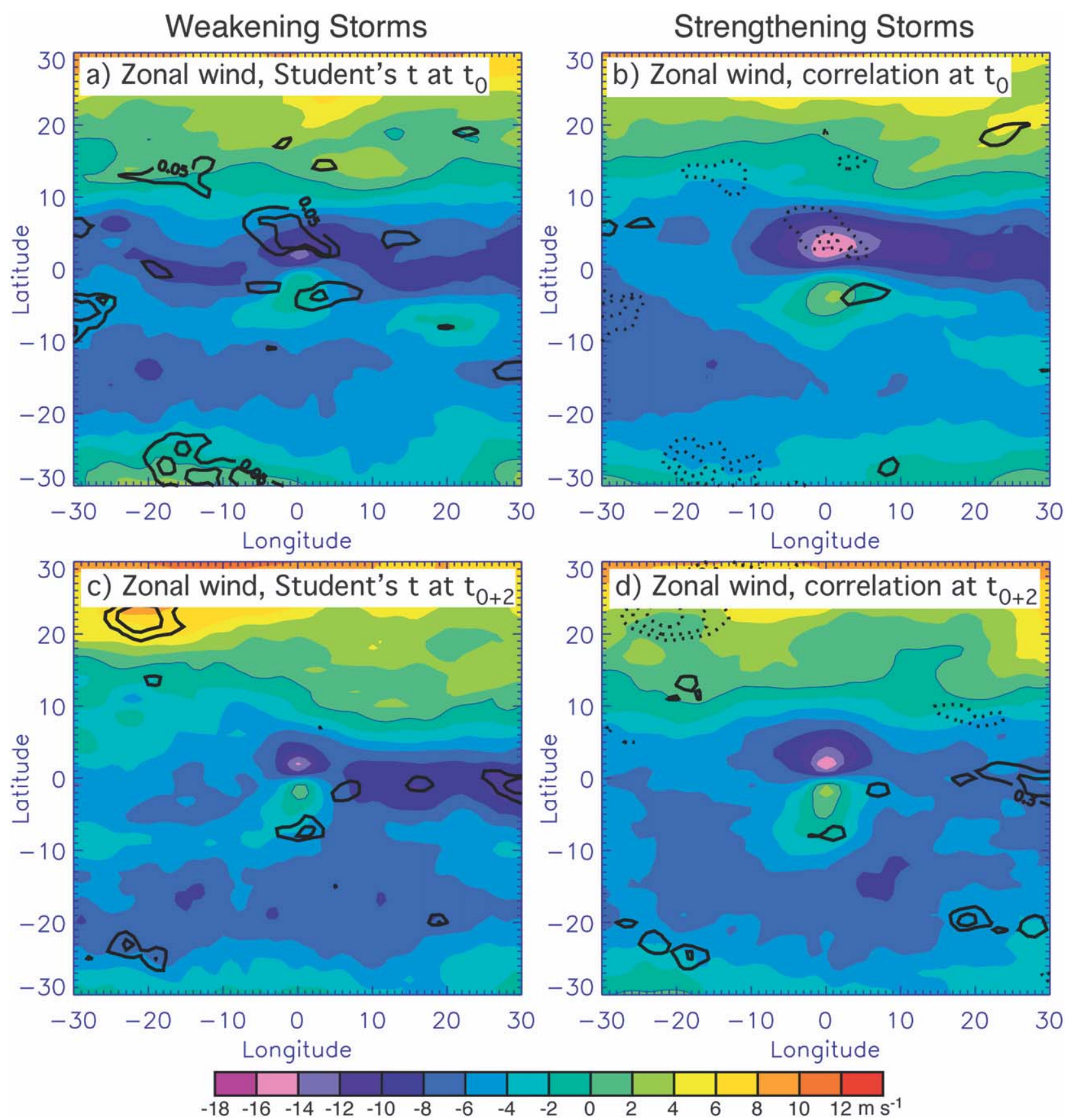

FIG. 12. GFS-derived fields for the composites over (left) weakening and (right) strengthening storms. Fields shown by shading are 700-hPa zonal velocity at time (top) $t_{0}$ and (bottom) $t_{0+2}$. Contours in the left panels show locations where differences between the two composites are significant at the $95 \%$ and $99 \%$ confidence levels from the Student's $t$ test. Contours in the right panels show the correlation between the intensity change between days 2 and 4 and the 700-hPa zonal velocity. Solid (dotted) contours show correlations $\geq 0.3(\leq-0.3)$ at 0.1 intervals.

days after genesis for storms that reached at least tropical storm strength. Indeed, the primary determinant of intensity change appears to be the relationship of the storm to the upper-tropospheric flow features, particularly those that constrain storm outflow and enhance vertical wind shear, in agreement with many previous studies (e.g., Rodgers and Gentry 1983; Holland and Merrill 1984; Merrill 1988; Molinari and Vollaro 1989; Rodgers et al. 1991; Hanley et al. 2001; Kimball and Evans 2002; Rappin et al. 2010). 

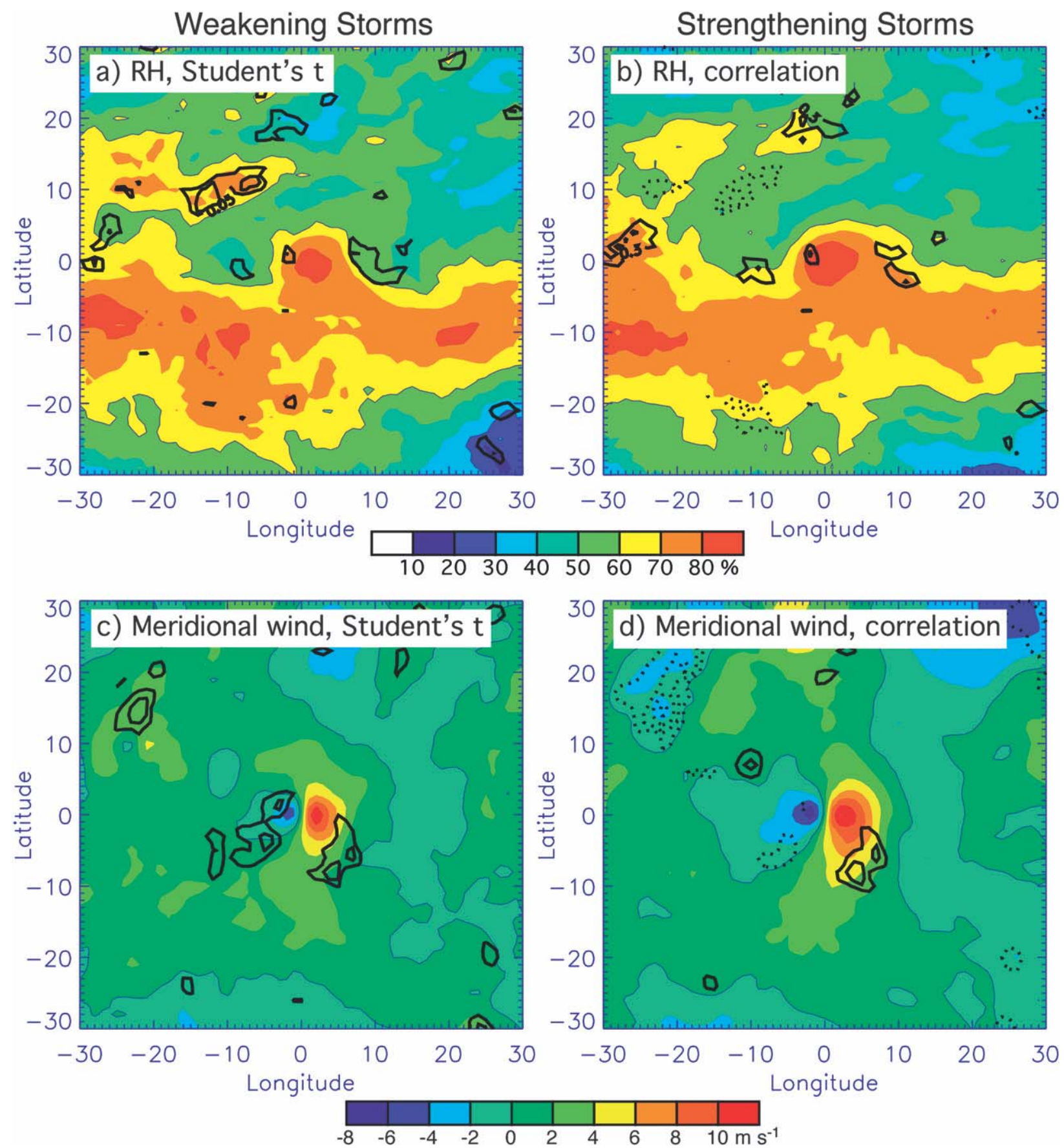

FIG. 13. GFS-derived fields for the composites over (left) weakening and (right) strengthening storms. Fields shown by shading are the 700-hPa (a),(b) RH and (c),(d) meridional velocity at time $t_{0+2}$. Contours in the left panels show locations where differences between the two composites are significant at the $95 \%$ and $99 \%$ confidence levels from the Student's $t$ test. Contours in the right panels show the correlation between the intensity change between days 2 and 4 and the (b) 700-hPa RH or (d) meridional velocity. Solid (dotted) contours show correlations $\geq 0.3(\leq-0.3)$ at 0.1 intervals.

\section{Discussion}

The results of this study disagree with a number of recent papers that had supposedly confirmed the negative impacts of the SAL on Atlantic tropical cyclones. In this section, we identify several key assumptions that are often made in these other studies that the results herein bring into question. 

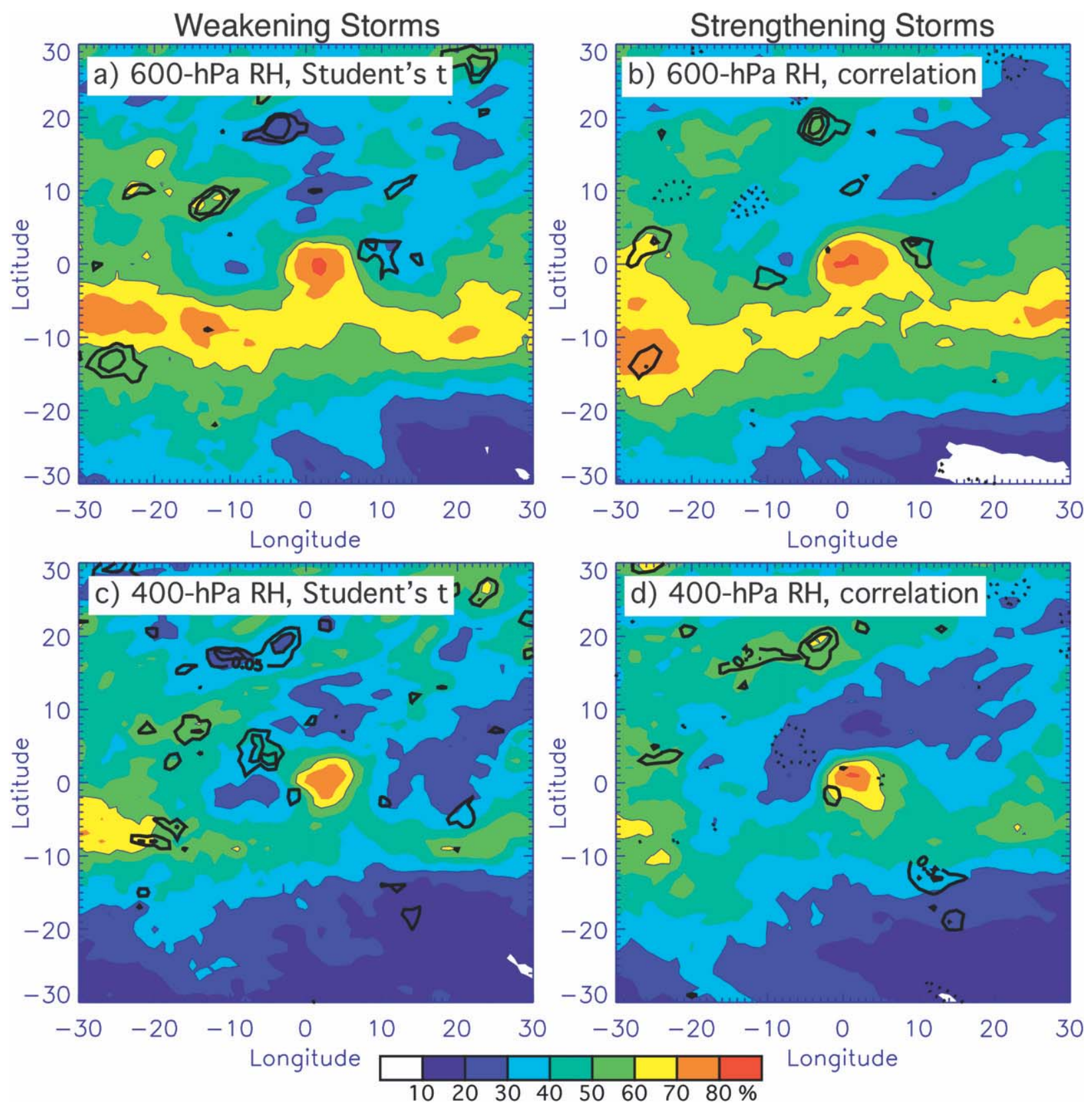

FIG. 14. GFS-derived fields for the composites over (left) weakening and (right) strengthening storms. Fields shown by shading are (top) 600- and (bottom) $400-\mathrm{hPa} \mathrm{RH}$ at $t_{0+2}$. Contours in the left panels show locations where differences between the two composites are significant at the $95 \%$ and $99 \%$ confidence levels from the Student's $t$ test. Contours in the right panels show the correlation between the intensity change between days 2 and 4 and the RH at the indicated level. Solid (dotted) contours show correlations $\geq 0.3$ ( $\leq-0.3$ ) at 0.1 intervals.

\section{a. Dry tropical air is $S A L$ air}

A frequent assumption made in many recent SAL papers (Jones et al. 2007; Dunion and Marron 2008; Reale et al. 2009; Shu and Wu 2009; Sun et al. 2008; Sun et al. 2009) is that very dry low- to midlevel tropical air is necessarily of Saharan origin. Dunion and Marron
(2008) separated soundings at four Caribbean sites into SAL and non-SAL categories, with the separation being accomplished based upon Geostationary Operational Environmental Satellite (GOES) multichannel SAL analyses (DV). It was assumed that the key sources of low- to midlevel dry air were SAL air masses and midlatitude dry-air intrusions; in other words, dry tropical 

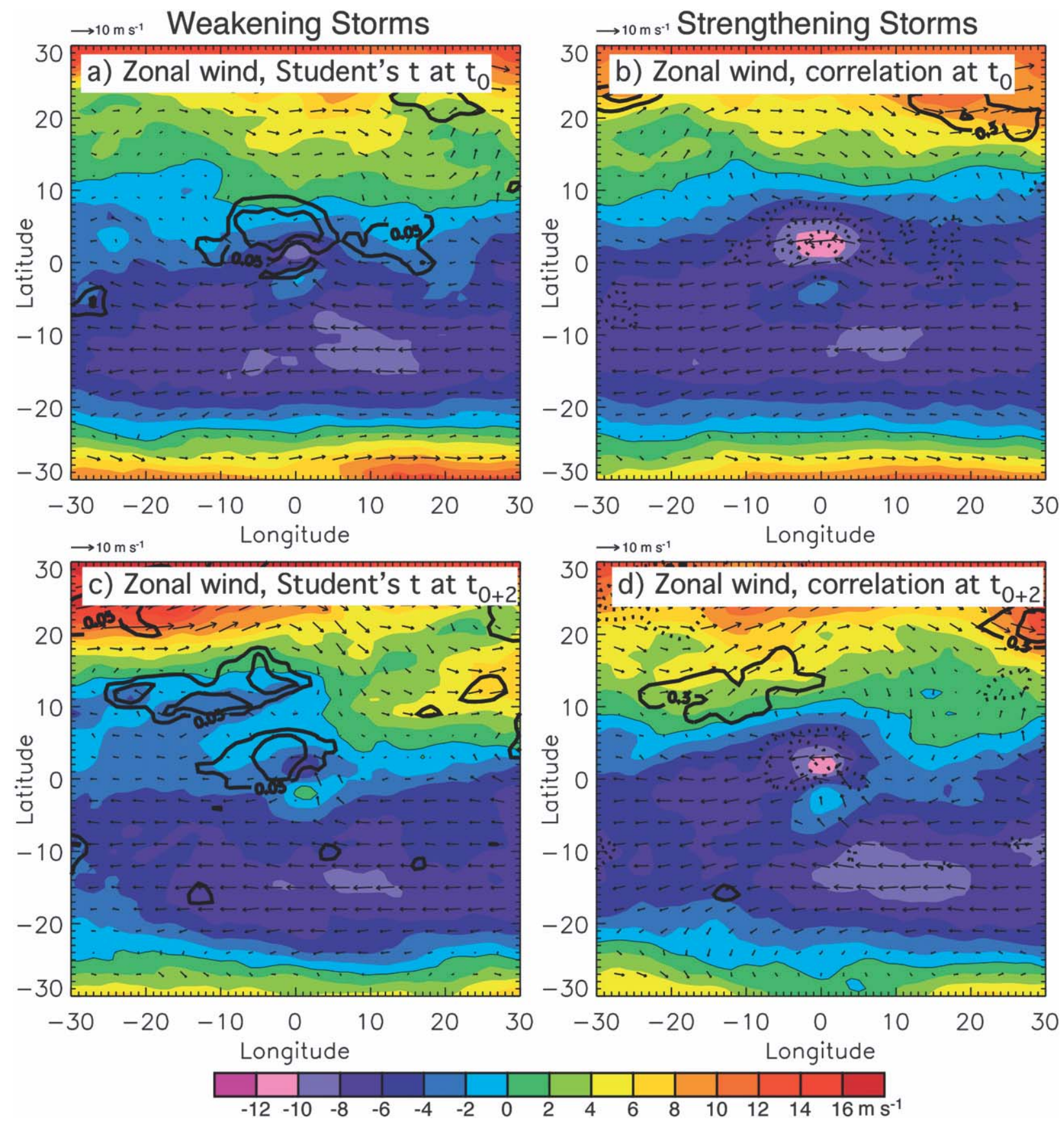

FIG. 15. GFS-derived fields for the composites over (left) weakening and (right) strengthening storms. Fields shown by shading are $400-\mathrm{hPa}$ zonal velocity at time (top) $t_{0}$ and (bottom) $t_{0+2}$. Contours in the left panels show locations where differences between the two composites are significant at the $95 \%$ and $99 \%$ confidence levels from the Student's $t$ test. Contours in the right panels show the correlation between the intensity change between days 2 and 4 and the 400 -hPa zonal velocity. Solid (dotted) contours show correlations $\geq 0.3$ $(\leq-0.3)$ at 0.1 intervals. Vectors indicate the mean $400-\mathrm{hPa}$ horizontal velocities.

air is SAL air. Other sources of dry tropical air such as regions of large-scale subsidence were not considered. This fact implies that their "SAL" sounding would be better labeled "dry tropical" since it has not been clearly demonstrated that the GOES data are able to clearly separate SAL sources of dry air from subsidence-driven sources.

A notable example of this problem of assuming that dry tropical air is necessarily of SAL origin is seen in Shu and $\mathrm{Wu}$ (2009). They showed AIRS data from Tropical 

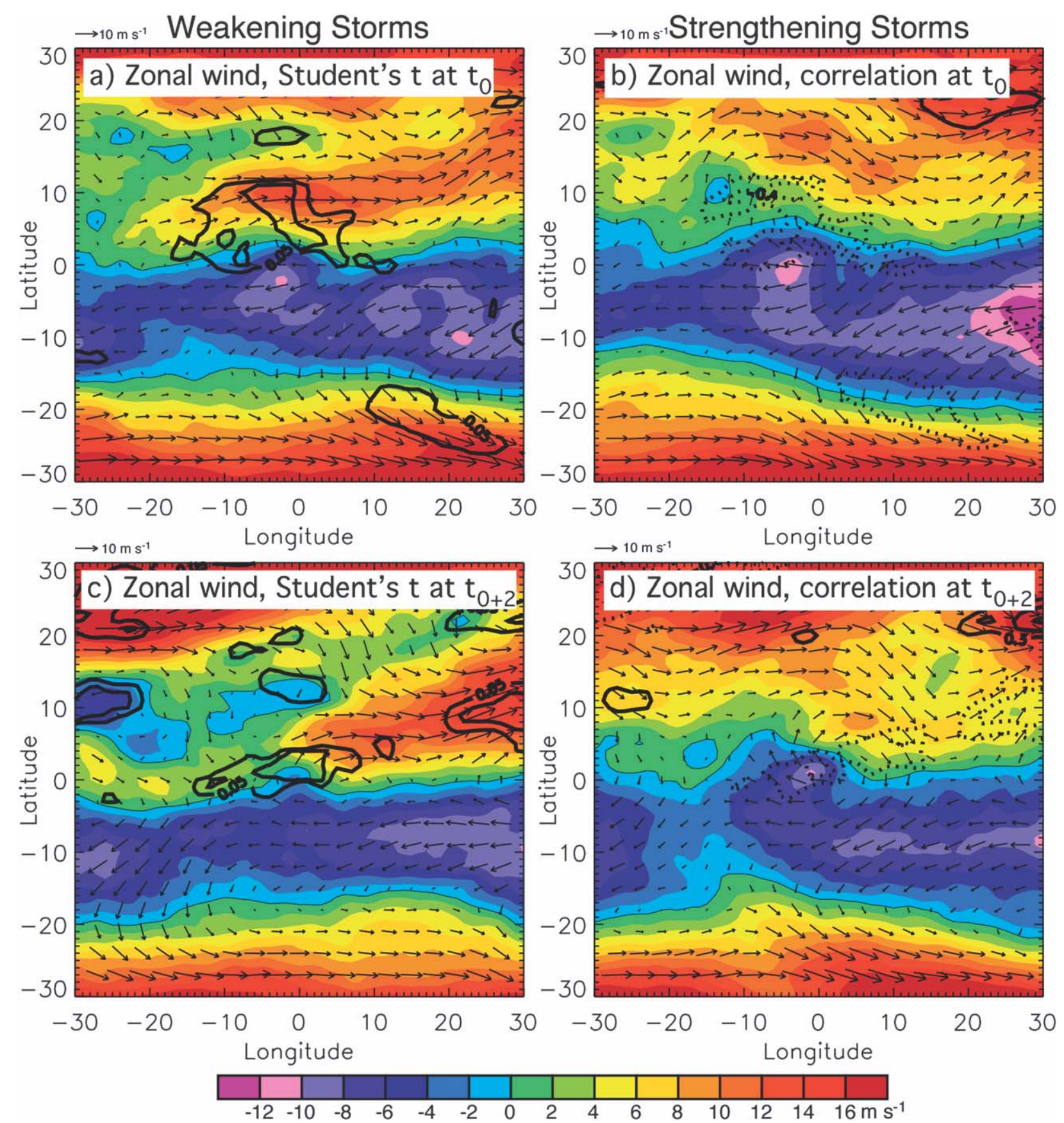

FIG. 16. As in Fig. 15 but for $200 \mathrm{hPa}$.

Storm Debby (2006) as an example of dry SAL air wrapping around the western side of Debby and intruding into the storm (see their Fig. 1). However, comparison of the AIRS data with MODIS AOD (Fig. 18) clearly shows that this dry tongue of air is virtually dust free [a similar pattern is seen in Reale et al. (2009) in a cyclonic system following Debby]. The main area of dust on the eastern side of Debby was associated with dry air $(<30 \%)$ in the
850-700-hPa layer (Fig. 18b), but with less dry air between 700 and 600 (>45\%; see Fig. 18c) and 600 and $500 \mathrm{hPa}$ (not shown). The very dry air ( $<15 \%$ humidity) to the north and west of Debby labeled SAL by Shu and $\mathrm{Wu}$ (2009) is dust free, and trajectories (not shown) computed from the GFS analyses confirm that the air is not of Saharan origin and is associated with substantial descent. In the 500-400-hPa layer (Fig. 18d), the extremely 


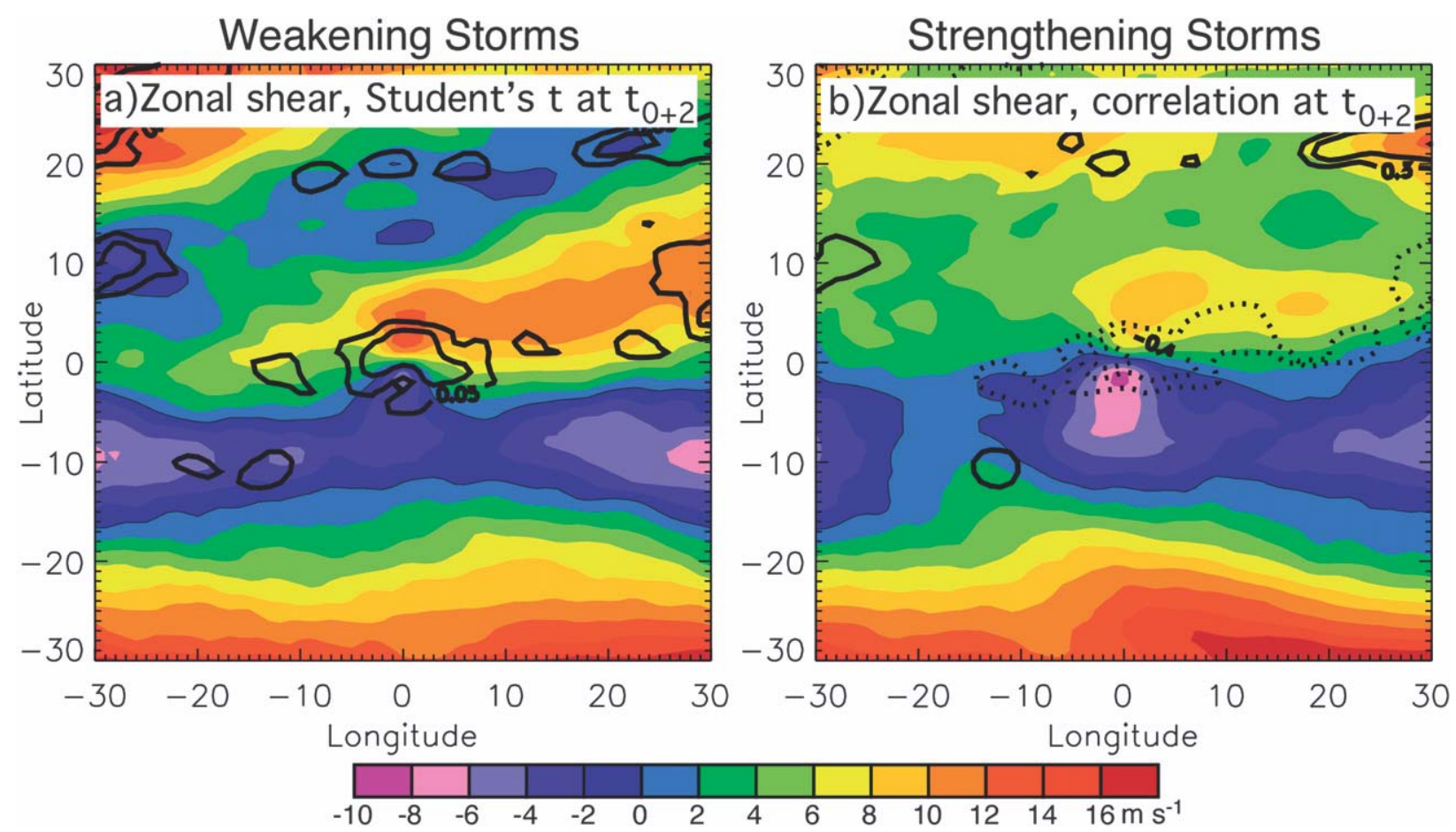

FIG. 17. GFS-derived fields for the composites over (left) weakening and (right) strengthening storms. Shown by shading is the vertical shear of the zonal wind between the 925-700- and 300-100-hPa layers at time $t_{0+2}$. Contours in the left panel show locations where differences between the two composites are significant at the $95 \%$ and $99 \%$ confidence levels from the Student's $t$ test. Contours in the right panel show the correlation between the intensity change between days 2 and 4 and the vertical shear. Solid (dotted) contours show correlations $\geq 0.3(\leq-0.3)$ at 0.1 intervals.

dry air north and west of Debby can still be seen in the layer above the SAL, further indicating that this dryness is of non-SAL origin.

\section{b. The SAL is dry throughout its depth}

The Dunion and Marron (2008) mean SAL sounding was characterized by a minimum in relative humidity at $500 \mathrm{hPa}$ and remained dry above that height. These results have led to assumptions in several studies (Jones et al. 2007; Wu 2007; Reale et al. 2009; Sun et al. 2008, 2009) that the SAL is very dry throughout its depth. As was shown in sections 3 and 4 , the SAL in the eastern Atlantic is often characterized by a midlevel layer of more elevated humidity (Carlson and Prospero 1972; Ismail et al. 2010; Messager et al. 2009). Furthermore, gradual net cooling of the SAL as it moves westward should lead to a rise in relative humidity at low levels. As a result, strict transport of the SAL by horizontal advection would imply a more moist midlevel layer and more moist low-level conditions in the western Atlantic than in the eastern Atlantic. Sun et al. (2008) attributed the decrease in Atlantic hurricane activity in 2007 compared to 2005 to increased influences of the SAL in 2007. Figure 2 in Sun et al. (2008) showed the June-July mean relative humidity for each year, and they attributed drier conditions at low- to midlevels in the western Atlantic to increased westward transport of dry SAL air. However, this dry air was situated near the bottom of a deep layer of very dry air that extended into the upper troposphere and was clearly not of SAL origin (similar results are found in Sun et al. 2009). Figure 3 in Sun et al. (2008) clearly shows the connection of this drier air to enhanced deep subsidence in the western Atlantic, with the largest drying in the middle to upper troposphere, suggesting a non-SAL mechanism.

\section{c. Guilt by proximity}

In many cases, attribution of storm weakening to the SAL is based upon the proximity of SAL air near the time of storm weakening rather than a clear demonstration of the direct impacts of the SAL (DV; Jones et al. 2007; Shu and Wu 2009). For example, in DV, the SAL was frequently implicated in intensity change based upon a subjectively determined proximity of SAL air (orange shading in their GOES SAL analyses), a priori assumptions that the GOES SAL analyses detected only the dry SAL air (and not other sources of dry tropical air), and that such proximity would necessarily 

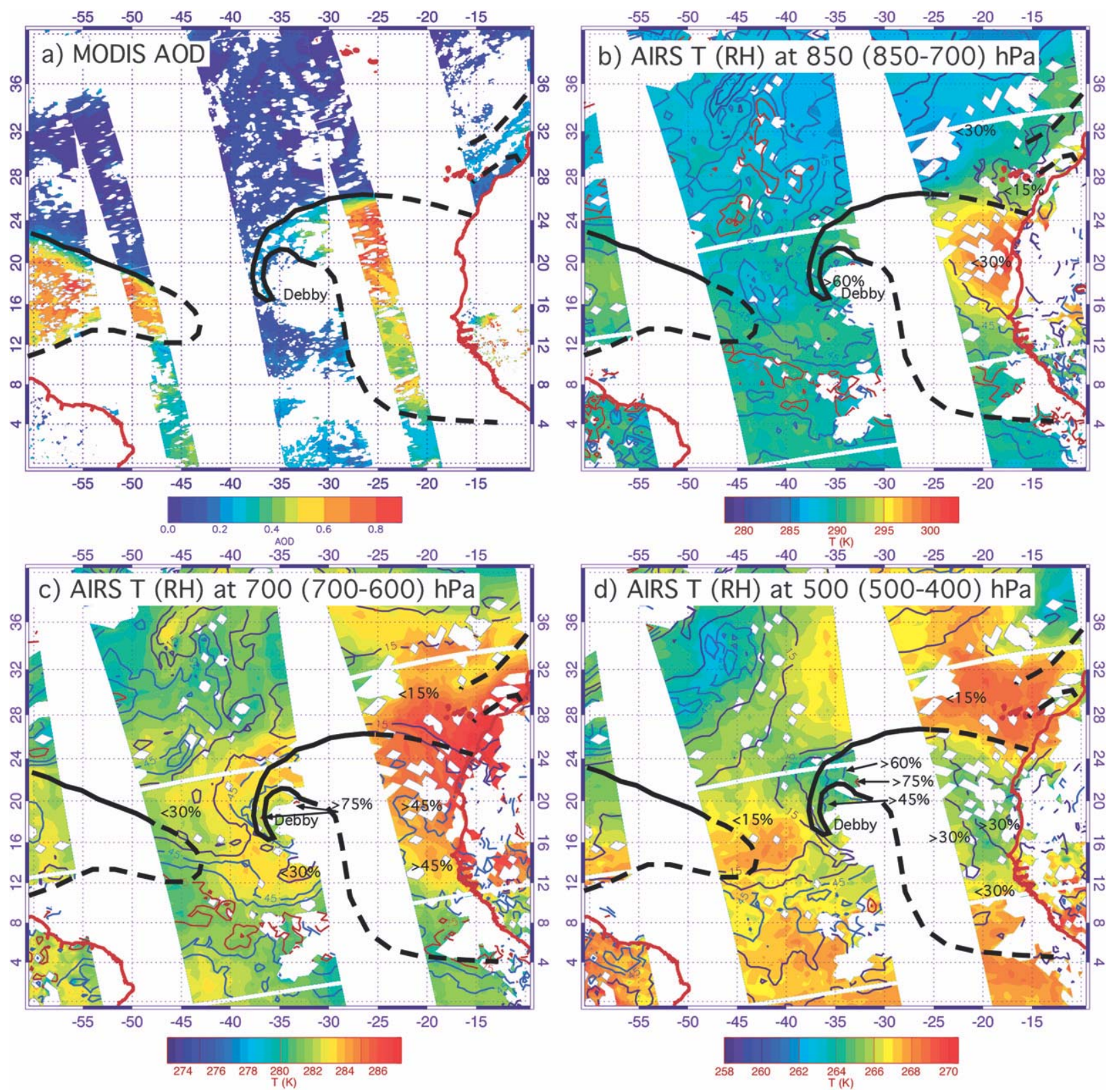

FIG. 18. (a) MODIS AODs from the Terra and Aqua level 2 orbit data. The black lines indicate the approximate boundaries of the dust layer and are dashed where the boundary location is uncertain. The location of Tropical Storm Debby is indicated. (b)-(d) AIRS-AMSU (level 2 orbit data) derived temperature (shading) and RH (contours) for the indicated levels. The contours are colored as follows: $15 \%$ and $30 \%$, purple; $45 \%$ and $60 \%$, blue; and $75 \%$ and $90 \%$, red.

weaken storms. The primary quantitative evidence provided was indirect, based upon forecast busts from the Statistical Hurricane Intensity Prediction Scheme (SHIPS), but alternative causes for intensity forecast errors were not explored.

Jones et al. (2007) examined the evolution of Hurricane Erin (2001), with a focus on its early stages when the storm struggled to intensify. They argued that the SAL likely suppressed convection, thereby inhibiting development. However, they showed little direct evidence for a SAL influence except for demonstrating the proximity of the SAL during this early stage. Very dry air aloft (in the 500-300-hPa layer) was incorrectly attributed to the SAL, but is clearly at heights typically above the SAL. They showed that Erin moved through a region of 5-day-averaged high aerosol optical depths, but the near proximity of SAL air does not necessarily imply ingestion of SAL air into the storm circulation. 
The composite analysis in section 5 suggests that the SAL is typically present even in intensifying storms, so proximity is clearly an insufficient justification for attributing weakening of a storm to the SAL.

\section{d. Assimilation of AIRS implies impacts of the SAL}

Wu et al. (2006) performed simulations of Hurricane Isabel both with and without assimilation of AIRS-retrieved profiles of temperature and humidity and found that assimilation of the AIRS data slowed the development of Isabel and prevented the formation of another system to its east. The assumption is that the assimilation of AIRS data improved the representation of the SAL and, thereby, its impacts on these storms. ${ }^{6}$ It is important to note that assimilation of the AIRS data changes the thermodynamic fields not only within the SAL, but also within the moist tropical air mass producing the convection. Consequently, the impacts of assimilation do not imply the impacts of the SAL, but also changes outside of the SAL that can affect the evolution of the simulated storm. Specifically, if assimilating AIRS data dries the moist tropical air mass, convection would likely be reduced, slowing the development of any tropical disturbance.

Sun et al. (2009) made similar assumptions regarding data impacts, that is, that weakening of a storm when assimilating AIRS data implies an impact of the SAL rather than changes elsewhere. For example, they attributed to the SAL the weakening of a disturbance to the east of Tropical Storm Florence (2006) in a simulation including AIRS data because a simulation without AIRS data produced a stronger cyclonic system. However, their Figs. 9 and 10 suggest very little difference in the SAL characteristics near this disturbance but marked differences in the position of Florence and the other cyclonic disturbance. Given the stochastic behavior of convection and its interaction with a tropical vortex, a conclusion that the differences in the simulations were the result of the SAL is not straightforward. As shown by Sippel and Zhang $(2008,2010)$, small perturbations in and around a weak tropical vortex can produce widely varying storm evolutions and intensities, suggesting that the limited data experiments in Sun et al. (2009) are insufficient for attributing the effects to the SAL.

\section{e. Other composite studies}

The composite results of this study disagree with the study of Shu and Wu (2009), in which the authors

\footnotetext{
${ }^{6}$ A problem with the Wu et al. study, discovered by this author (who was a coauthor on the $\mathrm{Wu}$ et al. paper) was that the AIRS moisture data had been assimilated as if the assigned height (the bottom of the layer) was the height of the midpoint of the moisture layer, resulting in the introduction of a dry bias.
}

examined the occurrence of the "nearest location of dry air" with respect to the storm center. They found that the dry air was often located closer to storms that weakened than those that strengthened, and assumed that this indicated a negative influence of the SAL. However, there were several major flaws in their analysis. 1) They assumed incorrectly that essentially all dry tropical air at midlevels was SAL (see discussion above and Fig. 18), 2) they used a poorly defined metric of SAL influence (nearest point location of dry air), and 3) in their comparison of strengthening and weakening storms, presumably at any stage of the life cycle, they assumed that the dry air was the likely cause of weakening rather than vertical shear, cooler SSTs, or other factors.

A composite study of AEWs by Hopsch et al. (2010) found results consistent with the results of the present study. They composited wave structures for developing and nondeveloping waves for the time of passage of AEWs at $15^{\circ} \mathrm{W}$ (the west coast of Africa) as well as 2 days before and after. They found that developing waves generally contained stronger initial absolute and potential vorticities at mid- and lower levels, were associated with stronger convection and better coupling of convection with the wave, and had more moist mid- to-upper level environments ahead (to the west). When developers were compared to the strongest 33 nondevelopers, Hopsch et al. found that the key differences were 1) in the phasing of convection with the wave as the waves moved into the Atlantic, with the convection maximum in the wave trough of developers but to the east of the trough axis in nondevelopers; and 2) drier air at middle to upper levels ahead of the nondevelopers. Although they note the southward advection of low potential vorticity air (likely associated with the SAL) ahead of the nondevelopers and a possible role of the SAL, they also emphasize the fact that the key differences in moisture were at levels generally above the SAL. These results are thus consistent with the present study in emphasizing the role of upper-level processes in determining the potential for storm development.

\section{Conclusions}

Previous studies on the impacts of the Saharan air layer on tropical cyclone genesis and intensification have yielded mixed results, with some studies (Karyampudi and Carlson 1988; Karyampudi et al. 1999; Karyampudi and Pierce 2002) suggesting that the SAL can have a positive influence on development and other studies (Dunion and Velden 2004; Jones et al. 2007; Shu and Wu 2009) suggesting that the SAL may be a negative influence. Dunion and Velden (2004) described several ways by which the SAL can inhibit tropical cyclone growth, including increased vertical wind shear associated with 
the African easterly jet, increased thermodynamic stability caused by the elevated warm layer, and impacts of dry midlevel air, particularly in terms of the production of cold downdrafts. Since the DV study, the SAL has been emphasized by some as a de facto negative influence on Atlantic tropical cyclogenesis and evolution (Lau and Kim 2007a,b; Jones et al. 2007; Wu 2007; Reale et al. 2009; Shu and Wu 2009; Sun et al. 2008, 2009). To determine whether this emphasis is warranted, in this study, we used NASA satellite remote sensing data and NCEP GFS analyses to evaluate the negative impacts proposed by DV. The conclusions below apply only to storm systems that developed into tropical storms and hurricanes. It is not known to what extent these results apply to nondeveloping storm systems.

Key findings are as follow:

- GFS wind fields suggest that enhanced low-level easterlies associated with the AEJ do not clearly produce inhibiting amounts of vertical wind shear near developing disturbances, but do usually provide significant background cyclonic vorticity for these disturbances. Near the time of storm formation, the AEJ is present in both strengthening and weakening storms and is typically as strong or slightly stronger in the strengthening cases prior to and at the time of formation. The only statistically significant difference appears around 2 days after formation when winds associated with the AEJ are about $2 \mathrm{~m} \mathrm{~s}^{-1}$ stronger to the east trailing the weakening storms. Whether these enhanced easterlies are a cause of weakening or a result of weak disturbance growth (the wave growing at the expense of the jet) cannot be determined. Bear in mind, though, that this finding is dependent upon the representation of the jet and hurricane vortex in the GFS analyses. Further research is required to verify this finding observationally and with high-resolution modeling.

- The warm SAL air is usually confined to areas north of developing storms (and the AEJ) such that higherstability air generally does not impact precipitating regions of storms that lie mostly south of the jet. In fact, the high stability in the SAL suppresses convection where it is not important for development and confines the convection to the cyclonic vorticity-rich side of the AEJ, where convection is most beneficial to development.

- Dry air in the tropical Atlantic is not uniquely a result of the Saharan region. Outside of the Atlantic ITCZ, the subtropical and eastern Atlantic regions are characteristically dry, with dry regions collocated with areas of large-scale descent on the eastern and equatorward sides of the Bermuda high pressure system (similar to other large oceanic basins). Drying caused by the Sahara is evident primarily at low levels (below the 700-600-hPa layer) over the Sahara, where warming from the surface reduces the relative humidity substantially. At midlevels ( $600-400 \mathrm{hPa})$, deep dry convective mixing over the Sahara results in a moistening and slight cooling effect relative to the extremely dry ambient air at the middle and upper levels in the region.

- A comparison between composite global model analyses for intensifying storms and weakening storms shows little evidence for a significant negative SAL impact. Differences in the distributions of CAPE and CIN are not statistically significant and the AEJ is actually as strong or stronger in cases of intensifying storms through the time of formation (see the first conclusion above). Differences in relative humidity are not significant until 2 days after formation, with weakening cases having an area of drier air just east of the storms. Although the enhanced dryness in the weakening cases may be related to the SAL, it may also be the result of the smaller and weaker vortex in the weakening cases, which would result in reduced northward transport of moist air to the east of the vortex. In addition, the dry air extended well above the SAL, suggesting a non-SAL source of the dry air. The key differences in the environmental conditions were in the upper-level zonal flow, particularly in terms of the constraints on storm outflow and the magnitude of the vertical wind shear. As above, these findings are dependent upon the representation of the SAL and hurricane vortex in the GFS analyses.

The results of this study suggest that the SAL has perhaps been overemphasized by some in the research community as a major negative influence on tropical cyclone genesis and evolution. In fact, the evidence appears to be more to the contrary in that the Sahara is the source of the AEJ, which acts as both a source of energy for AEWs and a source of strong background cyclonic vorticity, and there is evidence of a positive influence through an induced vertical circulation associated with the AEJ. To the extent that the SAL may be a negative influence on storm evolution, one must recognize that the SAL is just one of many factors influencing tropical cyclogenesis and evolution in the Atlantic. Each storm must be examined carefully within the context of the larger-scale wind and thermodynamic fields (either from global analyses or satellite data), particularly in terms of other sources of vertical wind shear and dry air (i.e., subsidence drying versus warming over the Sahara).

The impacts of African dust have not been evaluated here. Recent studies by Evan et al. $(2007,2008)$ and Lau and $\operatorname{Kim}(2007 \mathrm{a}, \mathrm{b})$ have suggested a link between dust activity and seasonal hurricane activity, although it is not 
yet clear whether this link is causative or merely correlative (Evan et al. 2007). The impacts of dust on microphysical processes and on hurricane intensity and evolution are even less clear, so caution should be taken before attributing to African dust a broad negative influence on seasonal hurricane activity or the development of individual storms.

Acknowledgments. The authors thank Karen Mohr and Ron McTaggart-Cowan for their helpful comments on the manuscript. This work was supported by Dr. Ramesh Kakar at NASA Headquarters with funds from the NASA Hurricane Science Research Program.

\section{REFERENCES}

Aumann, H. H., and Coauthors, 2003: AIRS/AMSU/HSB on the Aqua mission: Design, science objectives, data products, and processing systems. IEEE Trans. Geosci. Remote Sens., 41, 253-264.

Black, M. L., J. F. Gamache, F. D. Marks Jr., C. E. Samsury, and H. E. Willoughby, 2002: Eastern Pacific Hurricanes Jimena of 1991 and Olivia of 1994: The effect of vertical shear on structure and intensity. Mon. Wea. Rev., 130, 2291-2312.

Braun, S. A., and L. Wu, 2007: A numerical study of Hurricane Erin (2001). Part II: Shear and the organization of eyewall vertical motion. Mon. Wea. Rev., 135, 1179-1194.

—, M. T. Montgomery, and Z. Pu, 2006: High-resolution simulation of Hurricane Bonnie (1998). Part I: The organization of vertical motion. J. Atmos. Sci., 63, 19-42.

Burpee, R. W., 1972: The origin and structure of easterly waves in the lower troposphere of North Africa. J. Atmos. Sci., 29, $77-90$.

Carlson, T. N., and J. M. Prospero, 1972: The large-scale movement of Saharan air outbreaks over the northern equatorial Atlantic. J. Appl. Meteor., 11, 283-297.

Chan, J. C. L., K. S. Liu, S. E. Ching, and E. S. T. Lai, 2004: Asymmetric distribution of convection associated with tropical cyclones making landfall along the south China coast. Mon. Wea. Rev., 132, 2410-2420.

Chen, S. S., J. A. Knaff, and F. D. Marks Jr., 2006: Effects of vertical wind shear and storm motion on tropical cyclone rainfall asymmetries deduced from TRMM. Mon. Wea. Rev., 134, 3190-3208.

Cook, K. H., 1999: Generation of the African easterly jet and its role in determining West African precipitation. J. Climate, 12, $1165-1184$.

Corbosiero, K. L., and J. Molinari, 2002: The effects of vertical wind shear on the distribution of convection in tropical cyclones. Mon. Wea. Rev., 130, 2110-2123.

—, and —, 2003: The relationship between storm motion, vertical wind shear, and convective asymmetries in tropical cyclones. J. Atmos. Sci., 60, 366-376.

DeMaria, M., and J. Kaplan, 1994: A Statistical Hurricane Intensity Prediction Scheme (SHIPS) for the Atlantic basin. Wea. Forecasting, 9, 209-220.

, and _ 1999: An updated Statistical Hurricane Intensity Prediction Scheme (SHIPS) for the Atlantic and eastern North Pacific basins. Wea. Forecasting, 14, 326-337.
Diaz, H. F., T. N. Carlson, and J. M. Prospero, 1976: A study of the structure and dynamics of the Saharan air layer over the northern equatorial Atlantic during BOMEX. NOAA Tech. Memo. ERL WMPO-32, 61 pp.

Dunion, J. P., and C. S. Velden, 2004: The impact of the Saharan air layer on Atlantic tropical cyclone activity. Bull. Amer. Meteor. Soc., 84, 353-365.

— tropical sounding based on awareness of the Saharan air layer: Results from 2002. J. Climate, 21, 5242-5253.

Duvel, J. P., 1990: Convection over tropical Africa and the Atlantic Ocean during northern summer. Part II: Modulation by easterly waves. Mon. Wea. Rev., 118, 1855-1868.

Emanuel, K. A., 1989: The finite-amplitude nature of tropical cyclogenesis. J. Atmos. Sci., 46, 3431-3456.

Engelstaedter, S., I. Tegen, and R. Washington, 2006: North African dust emissions and transport. Earth Sci. Rev., 79, 73-100, doi:10.1016/j.earscirev.2006.06.004.

Evan, A. T., J. Dunion, J. A. Foley, A. K. Heidinger, and C. S. Velden, 2007: New evidence for a relationship between Atlantic tropical cyclone activity and African dust outbreaks. Geophys. Res. Lett., 33, L19813, doi:10.1029/2006GL026408.

—_, and Coauthors, 2008: Ocean temperature forcing by aerosols across the Atlantic tropical cyclone development region. Geochem. Geophys. Geosyst., 9, Q05V04, doi:10.1029/ $2007 \mathrm{GC} 001774$.

Frank, N. L., 1970: Atlantic tropical systems of 1969. Mon. Wea. Rev., 98, 307-314.

Frank, W. M., and E. A. Ritchie, 1999: Effects of environmental flow upon tropical cyclone structure. Mon. Wea. Rev., 127, 2044-2061. , and - 2001: Effects of vertical wind shear on the intensity and structure of numerically simulated hurricanes. Mon. Wea. Rev., 129, 2249-2269.

Franklin, J. L., S. J. Lord, S. E. Feuer, and F. D. Marks Jr., 1993: The kinematic structure of Hurricane Gloria (1985) determined from nested analyses of dropwindsonde and Doppler radar data. Mon. Wea. Rev., 121, 2433-2451.

Gray, W. M., 1968: Global view of the origin of tropical disturbances and storms. Mon. Wea. Rev., 96, 669-700.

Grist, J. P., 2002: Easterly waves over Africa. Part I: The seasonal cycle and contrasts between wet and dry years. Mon. Wea. Rev., 130, 197-211.

— , and S. E. Nicholson, 2001: A study of the dynamic factors influencing the rainfall variability in the West African Sahel. J. Climate, 14, 1337-1359.

Hanley, D., J. Molinari, and D. Keyser, 2001: A composite study of the interactions between tropical cyclones and uppertropospheric troughs. Mon. Wea. Rev., 129, 2570-2584.

Holland, G. J., and R. T. Merrill, 1984: On the dynamics of tropical cyclone structural changes. Quart. J. Roy. Meteor. Soc., 110, 723-745.

Hopsch, S. B., C. D. Thorncroft, and K. R. Tyle, 2010: Analysis of African easterly wave structures and their role in influencing tropical cyclogenesis. Mon. Wea. Rev., 138, 1057-1077.

Hsieh, J. S., and K. H. Cook, 2005: Generation of African easterly wave disturbances: Relationship to the African easterly jet. Mon. Wea. Rev., 133, 1311-1327.

Huffman, G. J., R. F. Adler, D. T. Bolvin, G. Gu, E. J. Nelkin, K. P. Bowman, E. F. Stocker, and D. B. Wolff, 2007: The TRMM multisatellite precipitation analysis: Quasi-global, multiyear, combined-sensor precipitation estimates at fine scale. J. Hydrometeor., 8, 38-55. 
Ismail, S., and Coauthors, 2010: LASE measurements of water vapor, aerosol, and cloud distributions in Saharan air layers and tropical disturbances. J. Atmos. Sci., 67, 1026-1047.

Jenkins, G. S., and A. Pratt, 2008: Saharan dust, lightning and tropical cyclones in the eastern tropical Atlantic during NAMMA-06. Geophys. Res. Lett., 35, L12804, doi:10.1029/ 2008GL033979.

,$- \ldots$, and A. Heymsfield, 2008: Possible linkages between Saharan dust and tropical cyclone rain band invigoration in the eastern Atlantic during NAMMA-06. Geophys. Res. Lett., 35, L08815, doi:10.1029/2008GL034072.

Jones, C., N. Mahowald, and C. Luo, 2004: Observational evidence of African desert dust intensification of easterly waves. Geophys. Res. Lett., 31, L17208, doi:10.1029/2004GL020107.

Jones, S. C., 1995: The evolution of vortices in vertical shear: Initially barotropic vortices. Quart. J. Roy. Meteor. Soc., 121, 821-851.

Jones, T. A., D. J. Cecil, and J. Dunion, 2007: The environmental and inner-core conditions governing the intensity of Hurricane Erin (2007). Wea. Forecasting, 22, 708-725.

Karyampudi, V. M., and T. N. Carlson, 1988: Analysis and numerical simulations of the Saharan air layer and its effect on easterly wave disturbances. J. Atmos. Sci., 45, 3102-3136.

haran air layer on tropical cyclogenesis over the eastern Atlantic. Mon. Wea. Rev., 130, 3100-3128.

— , and Coauthors, 1999: Validation of the Saharan dust plume conceptual model using lidar, Meteosat, and ECMWF data. Bull. Amer. Meteor. Soc., 80, 1045-1075.

Kimball, S. K., and J. L. Evans, 2002: Idealized numerical simulations of hurricane-trough interaction. Mon. Wea. Rev., 130, 2210-2227.

Landsea, C. W., and W. M. Gray, 1992: The strong association between western Sahelian monsoon rainfall and intense Atlantic hurricanes. J. Climate, 5, 435-453.

Lau, K. M., and J. M. Kim, 2007a: How nature foiled the 2006 hurricane forecasts. Eos, Trans. Amer. Geophys. Union, 88 (9), doi:10.1029/2007EO090002.

— Geophys. Res. Lett., 34, L23811, doi:10.1029/2007GL031538.

Marks, F., Jr., R. A. Houze Jr., and J. F. Gamache, 1992: Dualaircraft investigation of the inner core of Hurricane Norbert. Part I: Kinematic structure. J. Atmos. Sci., 49, 919-942.

Mass, C., 1979: A linear primitive equation model of African wave disturbances. J. Atmos. Sci., 36, 2075-2092.

Merrill, R. T., 1988: Environmental influences on hurricane intensification. J. Atmos. Sci., 45, 1678-1687.

Messager, C., D. J. Parker, O. Reitebuch, A. Agusti-Panareda, C. M. Taylor, and J. Cuesta, 2009: Structure and dynamics of the Saharan atmospheric boundary layer during the West African monsoon onset: Observations and analyses from the research flights of 14 and 17 July 2006. Quart. J. Roy. Meteor. Soc., 135, doi:10.1002/qj.469.

Molinari, J., and D. Vollaro, 1989: External influences on hurricane intensity. Part I: Outflow layer eddy angular momentum fluxes. J. Atmos. Sci., 46, 1093-1105.

Picon, L., and M. Desbois, 1990: Relation between METEOSAT water vapor radiance fields and large scale tropical circulation features. J. Climate, 3, 865-876.

Pierrehumbert, R. T., 1998: Lateral mixing as a source of subtropical water vapor. Geophys. Res. Lett., 25, 151-154.

Powell, M. D., 1990: Boundary layer structure and dynamics in outer hurricane rainbands. Part II: Downdraft modification and mixed-layer recovery. Mon. Wea. Rev., 118, 918-938.
Prospero, J. M., and T. N. Carlson, 1970: Radon-222 in the North Atlantic trade winds: Its relationship to dust transport from Africa. Science, 167, 974-977.

— over the western equatorial North Atlantic Ocean. J. Geophys. Res., 77, 5255-5265.

— Atlantic. Pure Appl. Geophys., 119, 677-691.

— E. Bonatti, C. Schubert, and T. N. Carlson, 1970: Dust in the Caribbean atmosphere traced to an African dust storm. Earth Planet. Sci. Lett., 9, 287-293.

Rappin, E. D., M. C. Morgan, and G. J. Tripoli, 2010: The impacts of an outflow environment on tropical cyclone intensification and structure. J. Atmos. Sci., in press.

Reale, O., W. K. Lau, K.-M. Kim, and E. Brin, 2009: Atlantic tropical cyclogenetic processes during SOP-3 NAMMA in the GEOS-5 global data assimilation and forecast system. J. Atmos. Sci., 66, 3563-3578.

Reasor, P. D., M. T. Montgomery, F. D. Marks Jr., and J. F. Gamache, 2000: Low-wavenumber structure and evolution of the hurricane inner core observed by airborne dual-Doppler radar. Mon. Wea. Rev., 128, 1653-1680.

Riehl, H., T. C. Yeh, J. S. Malkus, and N. E. La Seur, 1951: The northeast trade of the Pacific Ocean. Quart. J. Roy. Meteor. Soc., 77, 598-626.

Rodgers, E., and R. C. Gentry, 1983: Monitoring tropical cyclone intensity using environmental wind fields derived from short-interval satellite images. Mon. Wea. Rev., 111, 979-996.

_ , S. W. Chang, J. Stout, J. Steranka, and J.-J. Shi, 1991: Satellite observations of variations in tropical cyclone convection caused by upper-tropospheric troughs. J. Appl. Meteor., 30, 1163-1184.

Rogers, R., S. Chen, J. Tenerelli, and H. Willoughby, 2003: A numerical study of the impact of vertical shear on the distribution of rainfall in Hurricane Bonnie (1998). Mon. Wea. Rev., 131, 1577-1599.

Ross, R. S., and T. N. Krishnamurti, 2007: Low-level African easterly wave activity and its relation to Atlantic tropical cyclogenesis in 2001. Mon. Wea. Rev., 135, 3950-3964.

Salomonson, V. V., W. L. Barnes, P. W. Maymon, H. E. Montgomery, and H. Ostrow, 1989: MODIS: Advanced facility instrument for studies of the earth as a system. IEEE Trans. Geosci. Remote Sens., 27, 145-153.

Sherwood, S. C., 1996: Maintenance of the free-tropospheric tropical water vapor distribution. Part I: Clear regime budget. J. Climate, 9, 2903-2918.

Shu, S., and L. Wu, 2009: Analysis of the influence of the Saharan air layer on tropical cyclone intensity using AIRS/ Aqua data. Geophys. Res. Lett., 36, L09809, doi:10.1029/ 2009GL037634.

Sippel, J. A., and F. Zhang, 2008: A probabilistic analysis of the dynamics and predictability of tropical cyclogenesis. J. Atmos. Sci., 65, 3440-3459.

$\longrightarrow$, and -2010 : Factors affecting the predictability of Hurricane Humberto (2007). J. Atmos. Sci., in press.

Soden, B. J., 1998: Tracking upper tropospheric water vapor radiances: A satellite perspective. J. Geophys. Res., 103, 17 069-17 081.

_ vapor radiances in terms of layer-average relative humidities: Method and climatology for the upper, middle, and lower troposphere. J. Geophys. Res., 101, 9333-9343. 
Sultan, B., S. Janicot, and P. Drobinski, 2007: Characterization of the diurnal cycle of the West African monsoon around the monsoon onset. J. Climate, 20, 4014-4032.

Sun, D., K. M. Lau, and M. Kafatos, 2008: Contrasting the 2007 and 2005 hurricane seasons: Evidence of possible impacts of Saharan dry air and dust on tropical cyclone activity in the Atlantic basin. Geophys. Res. Lett., 35, L15405, doi:10.1029/ 2008 GL034529.

—, Z. Boybeyi, C. Yang, R. Yang, W. K. M. Lau, M. Kafatos, and G. Leptoukh, 2009: Numerical simulations of the impacts of the Saharan air layer on Atlantic tropical cyclone development. J. Climate, 22, 6230-6250.

Tao, W.-K., X. Li, A. Khain, T. Matsui, and S. Lang, 2007: Role of atmospheric aerosol concentration on deep convective precipitation: Cloud-resolving model simulations. J. Geophys. Res., 112, D24S18, doi:10.1029/2007JD008728.

Thorncroft, C. D., and B. J. Hoskins, 1994a: An idealized study of African easterly waves. I: A linear view. Quart. J. Roy. Meteor. Soc., 120, 953-982.

_ and - 1994b: An idealized study of African easterly waves. II: A nonlinear view. Quart. J. Roy. Meteor. Soc., 120, 983-1015.

, and K. Hodges, 2001: African easterly wave variability and its relationship to Atlantic tropical cyclone activity. J. Climate, 14, 1166-1179.
Tompkins, A. M., A. Diongue, D. J. Parker, and C. D. Thorncroft, 2005: The African easterly jet in the ECMWF Integrated Forecast System: 4DVar analysis. Quart. J. Roy. Meteor. Soc., 131, 2861-2885.

Wong, M. L. M., and J. C. L. Chan, 2004: Tropical cyclone intensity in vertical wind shear. J. Atmos. Sci., 61, 1859-1876.

Wong, S., and A. E. Dessler, 2005: Suppression of deep convection over the tropical North Atlantic by the Saharan air layer. Geophys. Res. Lett., 32, L09808, doi:10.1029/2004GL022295.

Wu, L., 2007: Impact of Saharan air layer on hurricane peak intensity. Geophys. Res. Lett., 34, L09802, doi:10.1029/2007GL029564.

— S. A. Braun, J. J. Qu, and X. Hao, 2006: Simulating the formation of Hurricane Isabel (2003) with AIRS data. Geophys. Res. Lett., 33, L04804, doi:10.1029/2005GL024665.

Zhang, C., and J. Pennington, 2004: African dry air outbreaks. J. Geophys. Res., 109, D20108, doi:10.1029/2003JD003978.

Zhang, H., G. M. McFarquhar, S. M. Saleeby, and W. R. Cotton, 2007: Impacts of Saharan dust as CCN on the evolution of an idealized tropical cyclone. Geophys. Res. Lett., 34, L14812, doi:10.1029/2007GL029876.

Zipser, E. J., and Coauthors, 2009: The Saharan air layer and the fate of African easterly waves-NASA's AMMA field study of tropical cyclogenesis. Bull. Amer. Meteor. Soc., 90, 1137-1156. 


\section{Popular Summary}

Reevaluating the role of Saharan air layer in atlantic tropical cyclogenesis and evolution Scott A. Braun, NASA/GSFC Submitted to the Monthly Weather Review

The existence of the Saharan air layer (SAL), a layer of warm, dry, dusty air that frequently moves westward off of the Saharan desert of Africa and over the tropical Atlantic Ocean, has long been appreciated. As air moves over the desert, it is strongly heated from below, producing a very hot air mass at low levels. Because there is no moisture source over the Sahara, the rise in temperature causes a sharp drop in relative humidity, thus drying the air. In addition, the warm air produces a very strong jet of easterly flow in the middle troposphere called the African easterly jet that is thought to play a critical role in hurricane formation. In recent years, there has been an increased focus on the impact that the SAL has on the formation and evolution of hurricanes in the Atlantic. However, the nature of its impact remains unclear, with some researchers arguing that the SAL amplifies hurricane development and with others arguing that it inhibits it. The argument for positively influencing hurricane development is based upon the fact that the African easterly jet provides an energy source for the waves that eventually form hurricanes and that it leads to rising motion south of the jet that favors the development of deep thunderstorm clouds. The potential negative impacts of the SAL include 1) low-level vertical wind shear associated with the African easterly jet; 2) warm SAL air aloft, which increases thermodynamic stability and suppresses cloud development; and 3) dry air, which produces cold downdrafts in precipitating regions, thereby removing energy needed for storm development. As part of this recent focus on the SAL and hurricanes (which motivated a 2006 NASA field experiment), there has been little emphasis on the SAL's potential positive influences and almost complete emphasis on its possible negative influences, almost to the point of claims that the SAL is the major suppressing influence on hurricanes in the Atlantic.

In this study, multiple NASA satellite data sets (TRMM, MODIS, CALIPSO, and AIRS/AMSU) and National Centers for Environmental Prediction global analyses are used to see if the proposed negative influences deserve all of the attention they have recently received. The results show that storms generally form on the southern side of the African jet, where favorable background rotation is high. The jet often helps to form the northern side of the storms and is typically stronger in storms that intensify than those that weaken, suggesting that jet-induced vertical wind shear is not a negative influence on developing storms. Warm SAL air is confined to regions north of the jet and generally does not impact the tropical cyclone precipitation south of the jet. A comparison of the environments of strongly strengthening storms and of weakening storms shows no differences in SAL structure, indicating that the SAL has little influence on whether storms weaken or intensify. The large-scale flow at upper levels above the SAL was found to be most important, with the environment of strengthening storms having very little vertical wind shear and also favoring more expansive outflow from the storm. The SAL is shown to occur in a large-scale environment that is already characteristically dry as a result of largescale subsidence (sinking air motions). Strong surface heating and deep dry convective mixing enhance dryness at low levels, but moisten the air at midlevels. Therefore, mid-to-upper-level dryness is not a defining characteristic of the SAL, but is instead a signature of subsidence. As a result, we conclude that the SAL is not the major negative influence on hurricanes that recent studies have emphasized. It is just one of many possible influences and can be both positive and negative. 